\title{
Perforated Fairings for Landing Gear Noise Control
}

\author{
K. Boorsma * and X. Zhang ${ }^{\dagger}$ \\ N. Molin ${ }^{\ddagger}$ \\ University of Southampton, UK \\ Airbus, Toulouse, France
}

\begin{abstract}
I. Abstract
Landing gears of commercial aircraft make an important contribution to total aircraft noise in the approach configuration. Using fairings to shield components from high speed impingement reduces noise. Furthermore, perforating these fairings has been confirmed by flight tests to further enable noise reduction. Following a more fundamental study on the application of perforated fairings, a study has been performed to investigate and optimize the benefits of bleeding air through landing gear fairings. By means of wind tunnel tests, an aerodynamic and acoustic survey has been performed on a simplified A340 look alike Main Landing Gear to explore the influence of (perforated) fairings on the lower part of the gear.

The results show that for this specific case, the application of impermeable fairings reduces noise in the mid- and high frequency range by shielding sharp edged components from high velocity impingement. However, below $1 \mathrm{kHz}$ the noise is shown to increase significantly. Application of the perforations is shown to diminish this low frequency increase whilst maintaining the reduction in the mid- and high frequency range. The aerodynamic and acoustic measurements point in the direction of the separated flow of the fairings interacting with the downstream gear components responsible for the low frequency noise increase. Bleeding of the air through the fairings reduces the large scale turbulence in the proximity of these components and hence diminishes the low frequency increase. A sharp noise increase is present in the ground view direction at the 500 $\mathrm{Hz}$ centred tertsband for the solid fairing, which is confirmed to originate from the articulation link fairing. The flow around the wheels remains largely unaltered by application of the fairings. Furthermore the application of fairings is shown to suppress vortex shedding from the lower part of the main leg.

A study on the effectiveness of perforation location reveals that the stagnation area perforations are responsible for most of the low frequency noise decrease. Both articulation link and undertray fairing contribute to the reduction. Exposing the perforate outside the stagnation area induces perforate self-noise, most significantly on the sides of the lower articulation link fairing. Agreeing with previous studies, the phenomenon scales with the local component of the shearing flow velocity and orifice diameter.
\end{abstract}

\section{Introduction}

A steady increase in the number of flights and airports has initiated research into aviation noise for commercial aircraft. A more stringent legislation and increased environmental awareness on this subject has led to an extra push. Research started with tackling the most dominant component, engine noise. As a result, engine noise has been reduced dramatically over the past decades,

${ }^{*}$ PhD Student, SES, University of Southampton, SO17 1BJ, UK.

${ }^{\dagger}$ Professor, SES, University of Southampton, SO17 1BJ, UK, Associate Fellow AIAA.

${ }^{\ddagger}$ Engineer, EEA Department, 316 Route de Bayonne, PO Box M0112/4, 31060 Toulouse, Cedex 03, France. 
thereby increasing relative importance of airframe noise for noise certification and environmental considerations.

Landing gear noise is the dominant component of airframe noise, especially for larger aircraft. ${ }^{1}$ Purely designed to perform their main function, landing gears feature an extremely aerodynamic unfriendly shape. The interaction of airflow with protrusions and cavities gives rise to unsteady flow phenomena constituting a potent sound generating mechanism.

Shielding landing gear components with fairings and thereby preventing this interaction has been considered before. ${ }^{1,2}$ High speed flow deflection onto other components and fairing self-noise are disadvantages of this measure. Bleeding air through the fairing results in a redistribution of the air flow. Flight tests using perforated fairings have been conducted before ${ }^{3}$ and demonstrated a noise decrease. The aim of the present research is to investigate and optimize the noise reduction potential by perforating these fairings.

The effect of bleeding air through a fairing on aeroacoustic performance of landing gear is a relatively new topic. Recent research on the subject ${ }^{4,5}$ has confirmed the flight test results, although the corresponding flow behavior has not been investigated. A previous study on perforated fairings employing a simplified bluff body geometry ${ }^{6}$ has shown that breakdown of the large scale flow structures associated with the fairings can be a major benefit, although one should be careful to prevent the adverse effect of perforate self-noise. Armed with the lessons learnt from this experiment, aerodynamics and acoustics of a simplified landing gear model have been investigated.

\section{Model and test set-up}

A visualization of the landing gear model is shown in Figure 1(a). It is a simplified 0.25

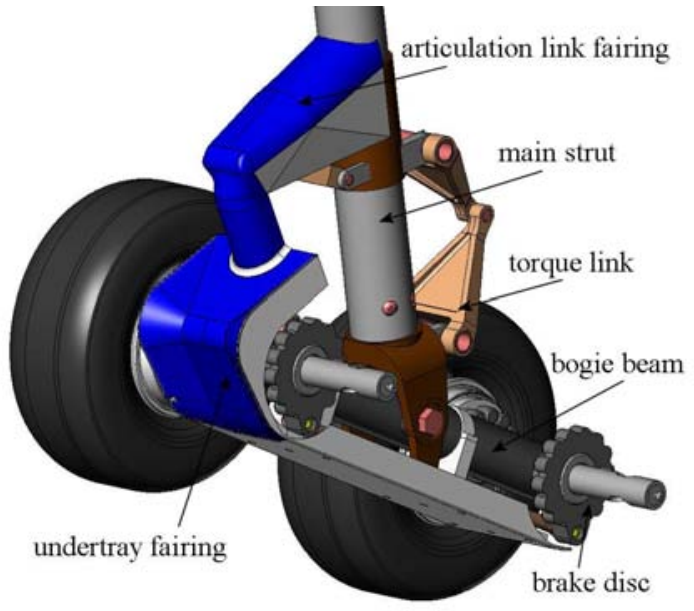

(a) Gear components (2 wheels omitted for clarity)

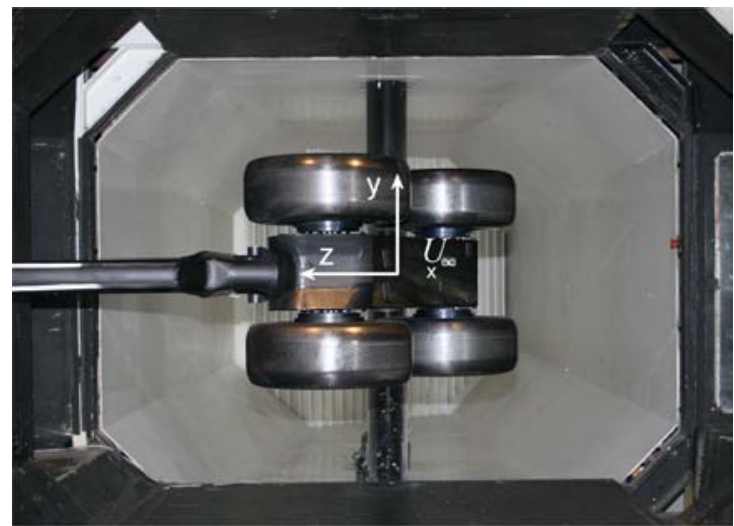

(b) Model as suspended in the tunnel

Figure 1. Visualization of the landing gear model.

scale model, based on the A340 Main Landing Gear. Any landing gear parts located above the articulation link are omitted, since the investigation concentrates on the articulation link and undertray fairing shielding articulation link and bogie beam respectively. Details on articulation, torque link and brake discs and links are accurately represented, although hoses are not present. Further more the outside rim of the wheels are covered with hub caps, and tyre thread is omitted.

Figure 1(b) shows the main strut inclined perpendicular to the flow and the tunnel side wall. A coordinate system can be defined originating in the center of the main strut-bogie beam junction. The $\mathrm{x}$-coordinate is in streamwise direction, positive pointing downstream. The $\mathrm{y}$ - and z-coordinate are defined in Figure 1(b).

The blue coloured part of the fairings are perforated with round holes resulting in an open area of $40 \%$. To discover the influence perforate location, tape has been applied to cover various parts of the perforated fairings. The three main configurations tested are without fairings, solid 
fairings (perforations taped) and perforated fairings indicated by LG, Solid and Perf respectively in the Figures.

Flow and acoustic measurements were performed in the closed circuit University of Southampton $7^{\prime} \times 5^{\prime}$ wind tunnel, using the $2.1 \mathrm{~m}$ wide, $1.7 \mathrm{~m}$ high octagonal cross section as depicted in Figure 1(b). The blockage amounts to $10 \%$ based on model frontal area (with fairings on). Freestream turbulence levels are approximately $0.2 \%$ over the velocity range considered, bounded by the maximum tunnel speed of $U_{\infty}=40 \mathrm{~m} / \mathrm{s}$. To monitor velocity scaling of aerodynamics and acoustics, tests are performed at $U_{\infty}=20, U_{\infty}=30$ and $U_{\infty}=40 \mathrm{~m} / \mathrm{s}$. An aircraft approach speed of $80 \mathrm{~m} / \mathrm{s}$ means that frequencies in the model scale test at $U_{\infty}=40 \mathrm{~m} / \mathrm{s}$ will be approximately twice the values of the ones for full scale flight.

For the aerodynamic tests, 3 different configuration have been investigated; the plain landing gear (no fairings), fairings applied with perforations taped and fairings applied with all perforations exposed indicated respectively by LG, Solid and Perf in the Figures.

\section{Experimental apparatus}

\section{IV.A. Oil flow visualization}

A mixture of titanium dioxide, paraffin and oleic acid was applied to the surfaces of the shell and strut. The oil is transported along surface streaklines leaving an image of the time-averaged flow field after it has dried up. Flow direction and separation are typical phenomenon observed with this technique. ${ }^{7}$

\section{IV.B. Particle Image Velocimetry}

Particle Image Velocimetry (PIV) was used to obtain information about the velocity field in the xz-plane at the model center $(\mathrm{y}=0)$. Measurements were performed using a Dantec FlowMap system. A $120 \mathrm{~mJ} \mathrm{Nd:YAG} \mathrm{dual-cavity} \mathrm{laser} \mathrm{was} \mathrm{fixed} \mathrm{on} \mathrm{a} \mathrm{table} \mathrm{to} \mathrm{the} \mathrm{side} \mathrm{of} \mathrm{the} 7^{\prime} \times 5^{\prime}$ tunnel, illuminating the relevant plane by firing through a small hole in the tunnel sidewall. A waterbased seed generator was used to seed the flow. An 80C60 HiSense CCD camera $(1280 \times 1024$ pixel resolution) was mounted above the tunnel, looking through a glass ceiling plate perpendicular to the laser sheet. The camera and laser were traversed in order to visualize the flow field upstream of the articulation link and the near wake downstream of the torque link and aft wheel. Using a $60 \mathrm{~mm}$ lens, the image size of the planes was around $120 \times 100 \mathrm{~mm}$. This resulted in 2 traverse positions of the camera to visualize the articulation link area and 7 traverse positions to visualize the near wake area with a decent overlap of around 15\%. 500 image pairs were recorded per configuration. Time between recordings was kept constant at $0.5 \mathrm{~s}$. The time between each pulse varied with tunnel speed in the order of $50 \mu \mathrm{s}$.

The images were post-processed using adaptive correlation with 2 refinement steps, starting with $128 \times 128$ and ending with $32 \times 32$ pixel interrogation area size. Vertical and horizontal overlap of the 2 images in each pair is set to $75 \%$. Erroneous vectors were removed using a range and peak validation.

\section{IV.B.1. Uncertainty}

The accuracy of the instantaneous velocity fields can be estimated by assuming an accuracy in the correlation of 0.1 pixel displacement. ${ }^{8}$ This corresponds to a maximum error in the velocity of 0.40 $\mathrm{m} / \mathrm{s}$. Using error analysis for multi-sample experiments as described by Moffat, ${ }^{9}$ the uncertainty in a time-averaged vector is $0.02 \mathrm{~m} / \mathrm{s}$.

\section{IV.C. Pressure tappings}

Static pressure was measured at various positions on the wheel surface. The centerline was tapped at $5^{\circ}$ increment on a $90^{\circ}$ section. Hence the wheel is rotated in 4 steps in order to get a $360^{\circ}$ centerline pressure distribution. Complimentary to the centerline tappings, the shoulder of the 
tyre surface was instrumented with 32 taps. The rotation of the wheel in steps of $90^{\circ}$ increment yields 4 locations for measurement of the shoulder taps. Since the model is inclined parallel to the streamwise xz-plane, left and right wheels should yield identical pressure distributions. Therefore only the pressures on the front and rear wheels located in the half space with positive y-coordinate (see Figure 1) are measured.

The total of 51 taps are connected to a Scanivalve ZOC22B/32PxX2 - 20' H20 pressure scanner via tubing inside the wheel. The ZOC module inside the wheel contains piezoresistive pressure sensors (capable of measuring up to 64 channels) and a pressure scanner temperature sensor. This data is then digitized in the RADBASE3200 and passed to the PC for temperature corrected unit conversion. Using the internal calibration, the system readily gives a pressure output and an individual calibration is not deemed necessary.

The normalized pressure coefficient can be defined as

$$
C p=\frac{p-p_{\infty}}{0.5 \rho U_{\infty}^{2}}
$$

where $p_{\infty}$ is the static pressure of the tunnel pitot tube and $\rho$ is derived from barometer and temperature readings in the tunnel using the ideal gas law. Since the reference pressure of the recording instrumentation is connected to the static pressure port of the tunnel pitot tube, the output is a measure for $p-p_{\infty}$.

In each frame, the channels are sampled at a rate of $50 \mu \mathrm{s}$ and averaged over 32 samples. For each run, 50 frames of data are acquired and averaged to obtain time averaged pressure. To minimize the influence of drifting of the signal, a zero run was performed before each measurement, which was subtracted before conversion to $C p$ values.

\section{IV.C.1. Uncertainty}

The error estimation by Moffat ${ }^{9}$ is used, which distinguishes fixed and variable sources, depending on whether the error it introduces is steady or changes during the time of one complete experiment. The fixed error consists of the accuracy of the data acquisition system and it's calibration. Scanivalve claims an accuracy of the current system of $\pm 0.12 \%$ of the full scale range, which converts into $\Delta C p= \pm 0.006$ at $U_{\infty}=40 \mathrm{~m} / \mathrm{s}$. The variable error is estimated by comparing the values of the 50 frames. The average standard deviation at $U_{\infty}=40 \mathrm{~m} / \mathrm{s}$ equals 0.013 , indicating an uncertainty of 0.002 for the 50 frames. Adding the fixed and variable errors by calculating the root-sum-square ${ }^{9}$ yields a total uncertainty of 0.006 at this velocity.

\section{IV.D. Unsteady pressure sensors}

Unsteady pressure sensors have been placed on various positions flush with the gear surface. For the present test, sensors have been placed in positions 1, 4 and 5 indicated in Figure 2. Additionally 2 sensors have been placed on the wheel centerline, spaced $\Delta \varphi=180^{\circ}$ apart. Both front and rear wheel are rotated in 4 steps to obtain unsteady centerline pressures around the wheel circumference spaced $\Delta \varphi=45^{\circ}$ apart using the 2 sensors.

The pressure sensors exhibit a reference tube which is connected to the static pressure from the tunnel pitot tube, enabling real time measurement of the fluctuating surface pressure. The sensors used are $2.4 \mathrm{~mm}$ diameter Kulite XCQ-093 sensors exhibiting a range of 0.35 bar and a natural frequency of $150 \mathrm{kHz}$. They are powered by a 8 channel VISHAY model 2150 strain gauge amplifier. Similar as for the microphones, analog to digital conversion was performed using a National Instruments' PXI-4472 24 bit data acquisition card, controlled by a PC using LabView software.

To convert the output voltage to pressure units, a calibration was performed using a Druck DPI 601-F pressure calibrator for each individual Kulite, assuming a linear relationship between voltage and pressure.

To minimize the influence of drifting of the signal, a zero run was performed before each measurement, which was subtracted before conversion to $C p$ values. The data were sampled at 


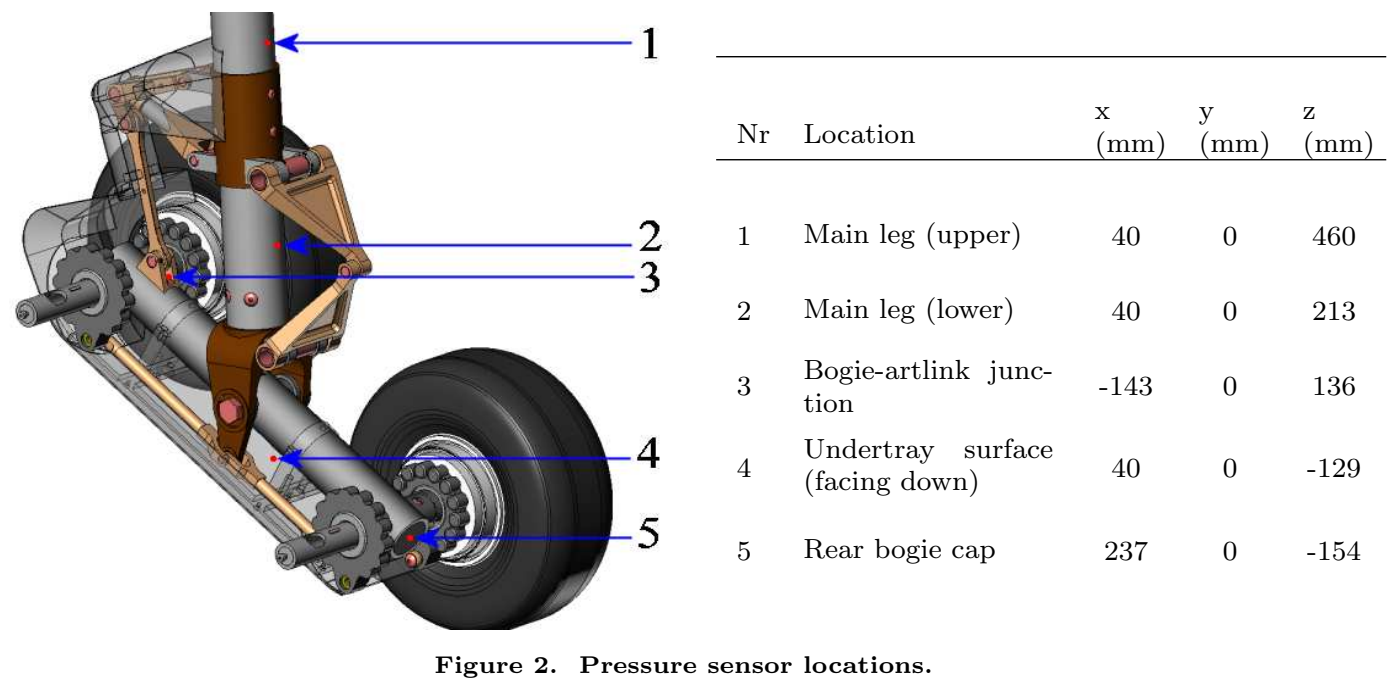

a frequency of $f=24 \mathrm{kHz}$ and a block size of $n=16384$, averaged over 100 blocks, giving a resolution of $\Delta f=1.46 \mathrm{~Hz}$. Equation 1 was used to convert the pressures to $C p$ values with input from barometer and temperature readings in the tunnel documented for each run.

\section{IV.D.1. Uncertainty}

The fixed error consists of the accuracy of the sensors and the calibration. The quoted accuracy of the calibrator is $\pm 0.05 \%$ of it's 0.5 bar range. The typical error of the Kulites due to combined non-linearity, hysteresis and repeatability is quoted at $0.1 \%$ of the 0.35 bar range. These combined figures add up to a fixed error of $\Delta C p= \pm 0.04$ at a freestream velocity of $U_{\infty}=40 \mathrm{~m} / \mathrm{s}$.

The variable error is estimated by comparing the average values of the 100 blocks. For the time averaged pressure, the average standard deviation at $U_{\infty}=40 \mathrm{~m} / \mathrm{s}$ equals 0.008 , indicating an uncertainty of 0.0008 for the 100 blocks. For the time averaged value of $C p$ at $U_{\infty}=40 \mathrm{~m} / \mathrm{s}$, this yields a total uncertainty of \pm 0.04 .

\section{IV.E. Microphones}

Various microphones have been placed on the model (flush with its surface). Referring to Figure 2, location $1,2,3$ and 5 have been instrumented.

The microphones used are Panasonic Omnidirectional Back Electret Condenser Cartridges, series WM-61A, with a response range of $20 \mathrm{~Hz}$ to $20 \mathrm{kHz}$. Since they are electret microphones, the signal was powered by preamplifiers, especially manufactured for this purpose. Analog to digital conversion was performed using a National Instruments' PXI-4472 data acquisition card, controlled by a PC using LabView software. A calibration factor was calculated using the recorded signal of a B\&K pistonphone (type 4230) to convert the raw data to narrowband spectra with a reference pressure $p_{\text {ref }}=2 \times 10^{-5} \mathrm{~Pa}$. A flat response over the frequency range is assumed. This assumption proved good enough for the present experiment after determination of the transfer function between a Panasonic and a calibrated 1/2" B\&K microphone, both exposed to white noise.

The data were sampled at a frequency of $f_{\text {sample }}=48 \mathrm{kHz}$ and a block size of $n=16384$, averaged over 60 blocks.

\section{IV.E.1. Uncertainty}

The accuracy of the microphone measurements consists of many parts and is hard to estimate. However microphone levels are predominantly compared between different configurations, thus the fixed error is unimportant. 
The variable error is estimated by comparing the values of the 60 acquired blocks and adds up to $0.5 \mathrm{~dB}$ for both sets of microphones, roughly constant for each frequency band.

\section{IV.F. Phased microphone array}

Two similar phased arrays are positioned in the starboard side wall and the ceiling of the $7^{\prime} \times 5^{\prime}$ wind tunnel. They both consist of 56 microphones, spirally placed in a wooden board with a diameter of $700 \mathrm{~mm}$, flush with its surface. The perpendicular distance between array and model

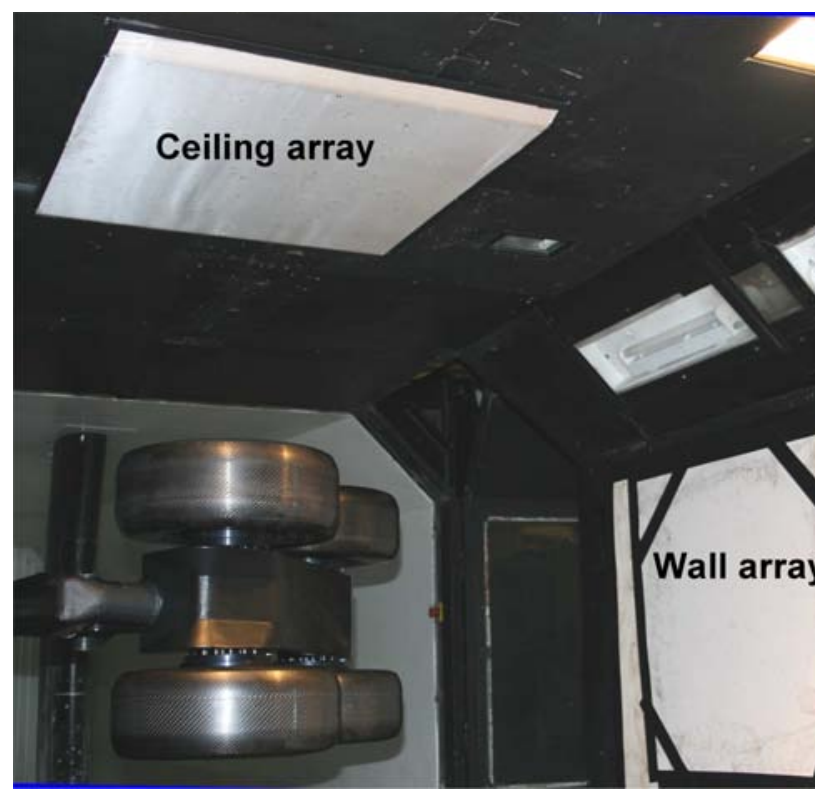

Figure 3. Set-up of microphone arrays in the $7^{\prime} \times 5^{\prime}$ tunnel.

center is $0.73 \mathrm{~m}$ for the ceiling array and $1.18 \mathrm{~m}$ for the side wall array. The different distance of the arrays to the model results in different resolution of the beamforming plot. Since the aperture of both arrays is the same and resolution varies linearly with distance, the side wall array exhibits a $61 \%$ better resolution than the ceiling array. Powering for the arrays is identical to the microphones as described in section IV.E. The same holds for the data acquisition, which was done simultaneously for both arrays. However sampling frequency is set to $f=48 \mathrm{kHz}$, block size $n=8192$ and the data were averaged over 120 blocks.

The beamforming code for the present experiments was written by Fenech ${ }^{10}$ and is based on conventional frequency-domain beamforming. 1/3-Octave band averaged beamforming plots were calculated for comparison between configurations, however the finite aperture of the array prevented sufficient resolution for localization below $2 \mathrm{kHz}$.

The side view beamforming plots from the ceiling array are aligned in the xz-plane at $\mathrm{y}=0$. However, the ground view plots from the side wall array are aligned with the bogie beam and rotated by $33^{\circ}$ going through the model origin. For the previously mentioned integration of the beamforming plots, squared pressures are summed between $x= \pm 0.2 \mathrm{~m}$ and $z=-0.1 \mathrm{~m}$ to $z=0.3$ $\mathrm{m}$ for the ceiling array and between $x=-0.4 \mathrm{~m}$ to $x=0.3 \mathrm{~m}$ and $y= \pm 0.15 \mathrm{~m}$ for the side wall array.

\section{IV.F.1. Uncertainty}

The fixed error of the beamforming plots and their integrated levels is not relevant since they are only used for a comparison between configurations. A rough estimate for the variable error is obtained from a comparison between two identical configurations. This yields a maximum uncertainty of $1.5 \mathrm{~dB}$ for the beamforming plots and $0.45 \mathrm{~dB}$ for the integrated levels. 


\section{Results and discussion}

The results are divided into sections. Firstly results of the aerodynamic measurements are discussed, starting with flow around the fairings, near wake flow and flow around the wheels. Then acoustics are highlighted including microphone array and on-surface microphone measurements.

\section{V.A. Fairing Flow}

This section describes time-averaged flow features on the fairings. A front view of oil flow visualization on undertray and articulation link fairing is shown in Figure 4. The undertray stagnation
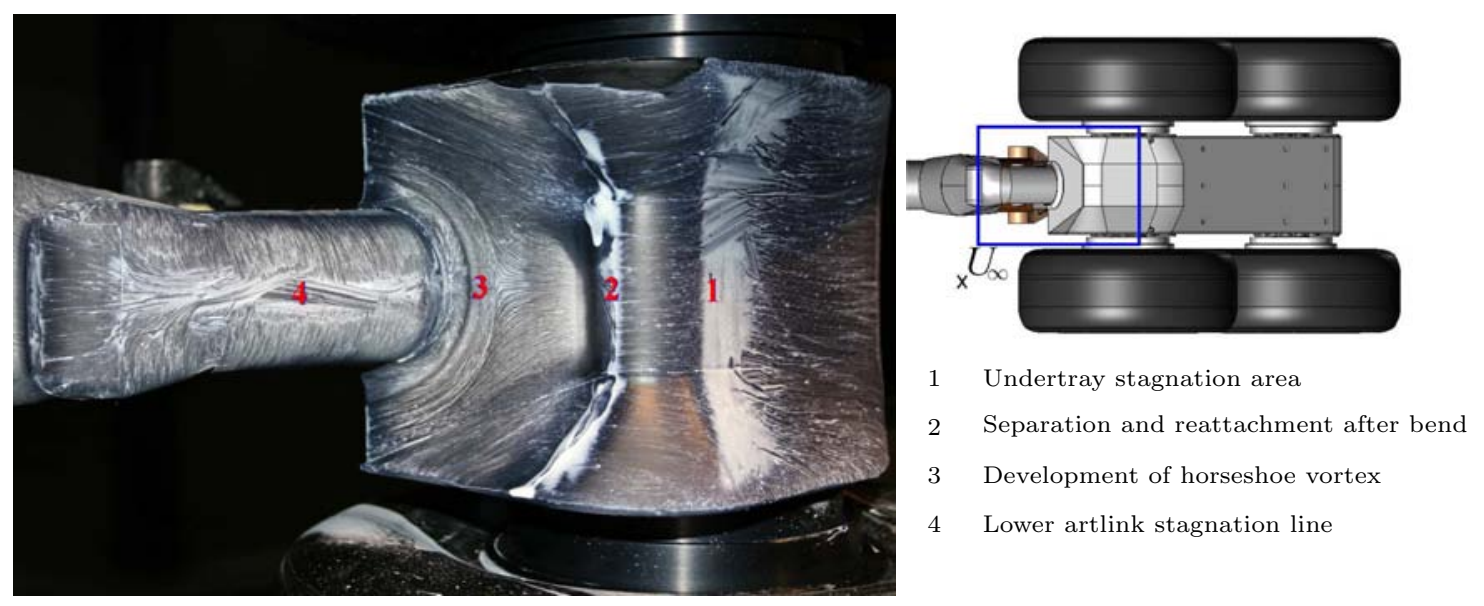

Figure 4. Front view of flow visualization of solid fairings $\left(U_{\infty}=40 \mathrm{~m} / \mathrm{s}\right)$.

line divides the flow in upward and downward directions (z-direction). The upward deflected flow impinges on the lower articulation link fairing and forms a horseshoe vortex around it. The lower articulation link itself divides the flow in transverse direction (y-direction), where it separates from the lower articulation sides as depicted in Figure 5. The streamlines on the upper articulation link indicate attached flow until the transition to the main leg is reached.

PIV is used to visualize the flow directly upstream of the articulation link fairing on the model centerline $(y=0)$ in Figure 6 . The effect of the fairing is to deflect the flow more in upward direction, since it's cross sectional frontal area is large compared to the bare articulation link. The impact of perforations is relatively small. Noticeable is the increase in velocity in the stagnation point area between $\mathrm{z}=320 \mathrm{~mm}$ and $\mathrm{z}=380 \mathrm{~mm}$ caused by the bleeding of the air. However, flow direction pattern remains largely unaltered, indicating that more porosity is needed if reduction of upward deflected velocities is desired.

Figure 7 visualizes the ground view of the downward directed flow by the undertray. The flow remains attached to the fairing surface until the trailing edge apart from the laminar separation bubble at the sharp bend close to the stagnation line. Instead of leaving the undertray at the trailing edge, part of the flow escapes sideways between the wheels as visualized in Figure 7. After reaching the undertray side, a side edge vortex forms of which the path is visible in the figure. An unsteady pressure sensor is positioned flush with the undertray surface at location 4 (see Figure 2). The flow visualization readily revealed attached flow in this region. Hence large scale fluctuations are not present and pressure spectra are dominated by electronic noise and therefore omitted. The 

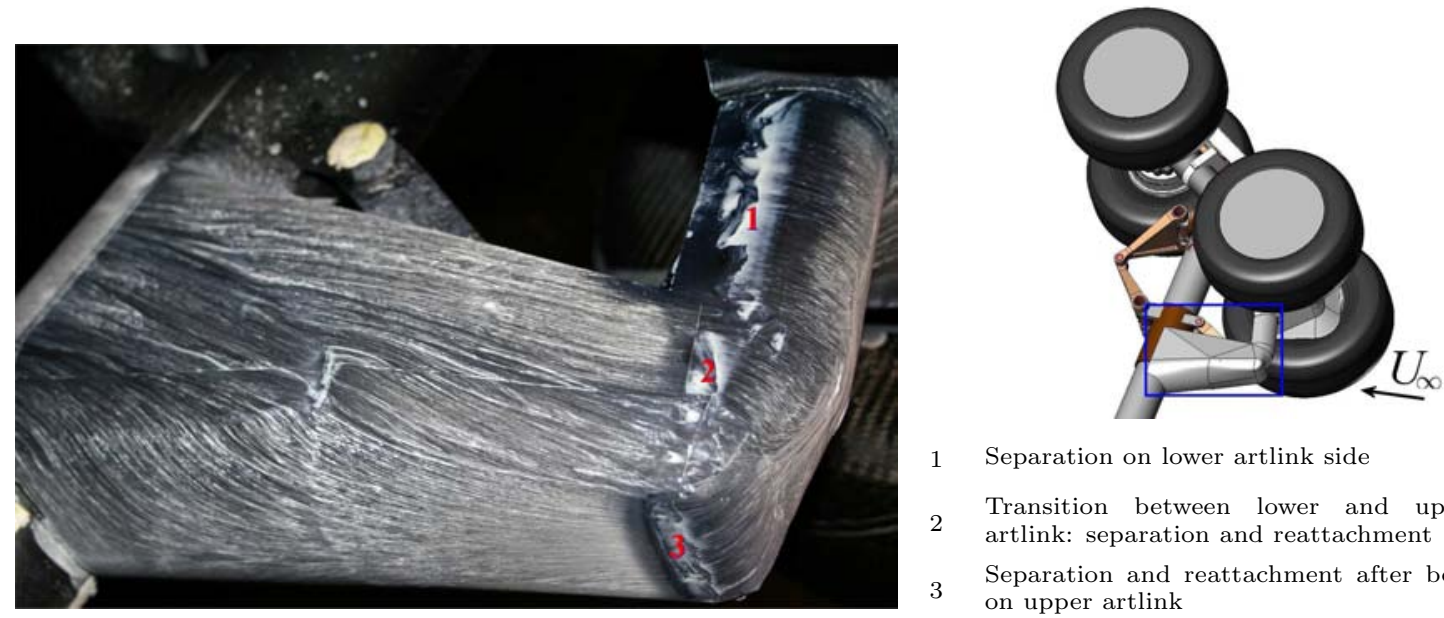

Separation on lower artlink side

Transition between lower and upper artlink: separation and reattachment

Separation and reattachment after bend on upper artlink

Figure 5. Side view of flow visualization of solid fairings $\left(U_{\infty}=40 \mathrm{~m} / \mathbf{s}\right)$.

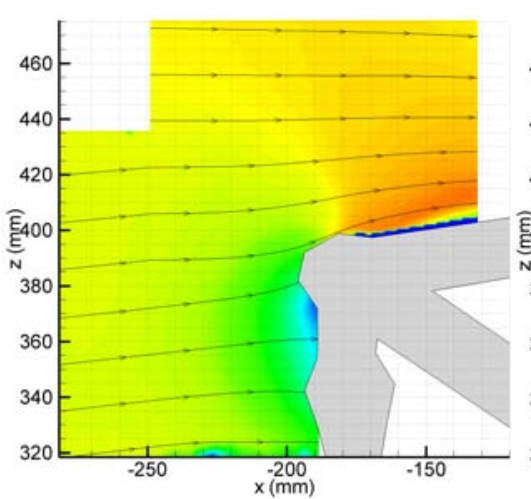

(a) LG

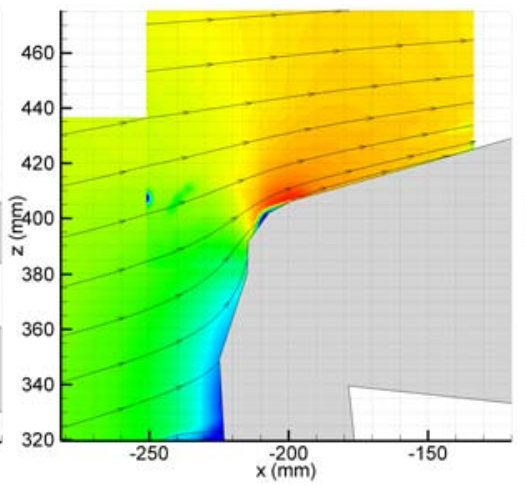

(b) Solid

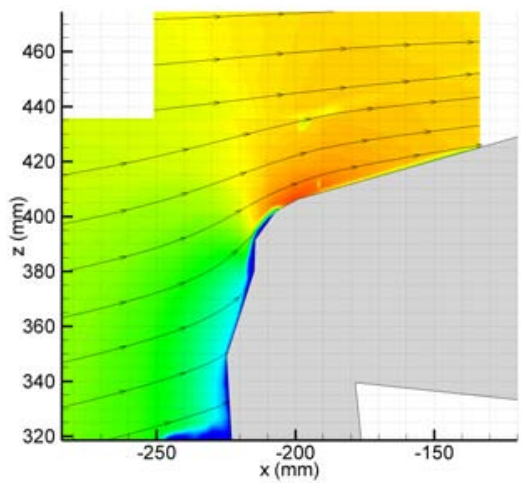

(c) Perf

Figure 6. Time-averaged velocity contours in the $\mathrm{xz}-$ plane at $\mathbf{y}=\mathbf{0}$ (articulation link area, $U_{\infty}=40 \mathrm{~m} / \mathrm{s}$ ). 

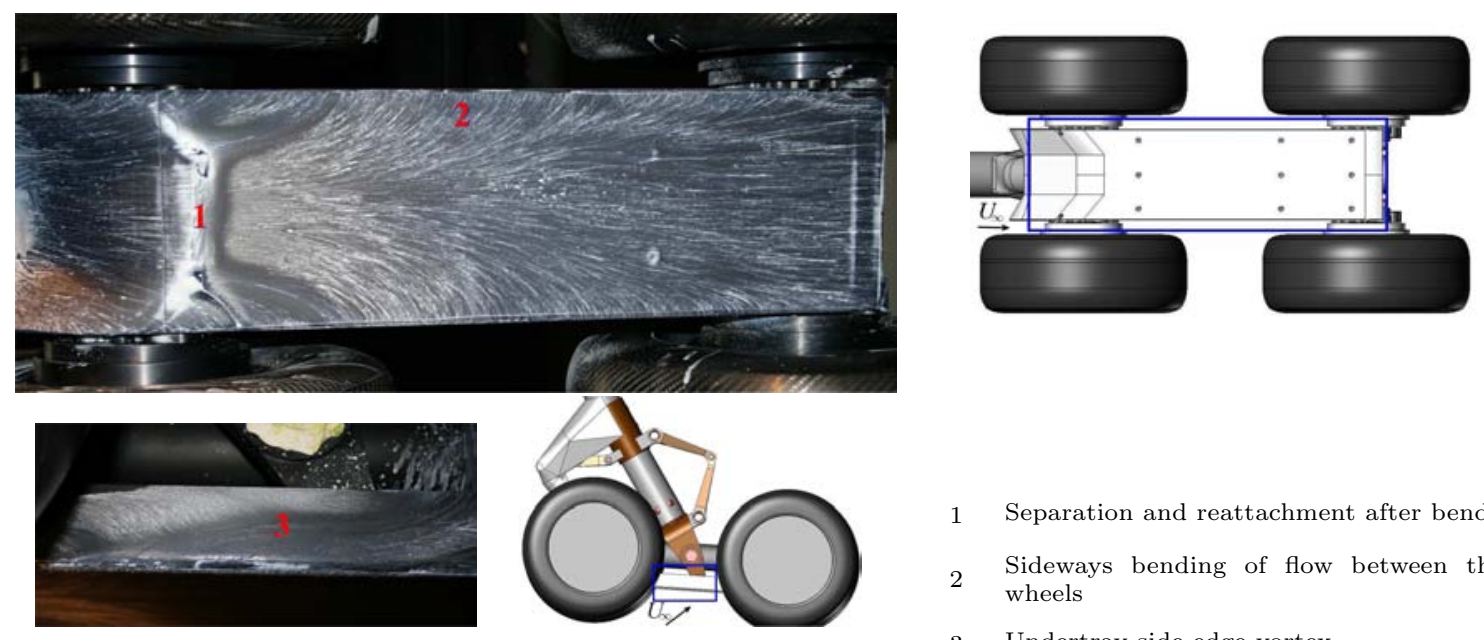

Separation and reattachment after bend

Sideways bending of flow between the wheels

3 Undertray side edge vortex

Figure 7. Ground view of flow visualization of solid fairings $\left(U_{\infty}=40 \mathrm{~m} / \mathbf{s}\right)$.

perforated and solid fairing configuration yield the same result, amounting to a mean pressure of $C p=0.22$ at this location.

\section{V.B. Near Wake Flow}

The flow conditions directly downstream of the gear (torque link, main leg and rear bogie area) have been investigated using PIV and unsteady pressure sensors. Time averaged velocity contours in the model centerplane are shown in Figure 8. As noticed before, the flow above $\mathrm{z}=340 \mathrm{~mm}$ in Figure 6(a) is not deflected upwards by the fairing, which results in a high streamwise velocity region in the top of Figure 8(a). The vectors illustrate that without fairings applied, the dominant wake flow direction is streamwise. An area of relatively high speed flow $\left(0.7 U_{\infty}\right)$ emerges between $\mathrm{z}=-100$ and $\mathrm{z}=50 \mathrm{~mm}$, resulting from the flow penetrating through the gear between the bogie beam and wheels. Application of the fairings alters the wake structure significantly. From the torque link junction, the flow is directed in upward and downward direction. Apparently deflection of the air in z-direction by the fairings results in spanwise flow features in the wake. Since the undertray fairing prevents the air from penetrating through the gear, the high speed velocity region between $\mathrm{z}=-100$ and $\mathrm{z}=50 \mathrm{~mm}$ partly disappears. Additionally, a dead flow area emerges below $\mathrm{z}=0 \mathrm{~mm}$. The flow that manages to escape in streamwise direction from the undertay side edge (Figure 7) results in a small area of high speed streamwise flow between $z=0$ and $z=50 \mathrm{~mm}$.

The corresponding contours of standard deviation are shown in Figure 9. As expected, application of fairings yields very low values of $u^{\prime}$ in the dead flow area below $\mathrm{z}=0 \mathrm{~mm}$. Apart from the dead flow area, the near wake generally exhibits lower values of $u^{\prime}$ without fairings applied. An explanation could be that the larger size of the fairings compared to the plain gear introduces large scale fluctuations and therefore higher values of $u^{\prime}$. 


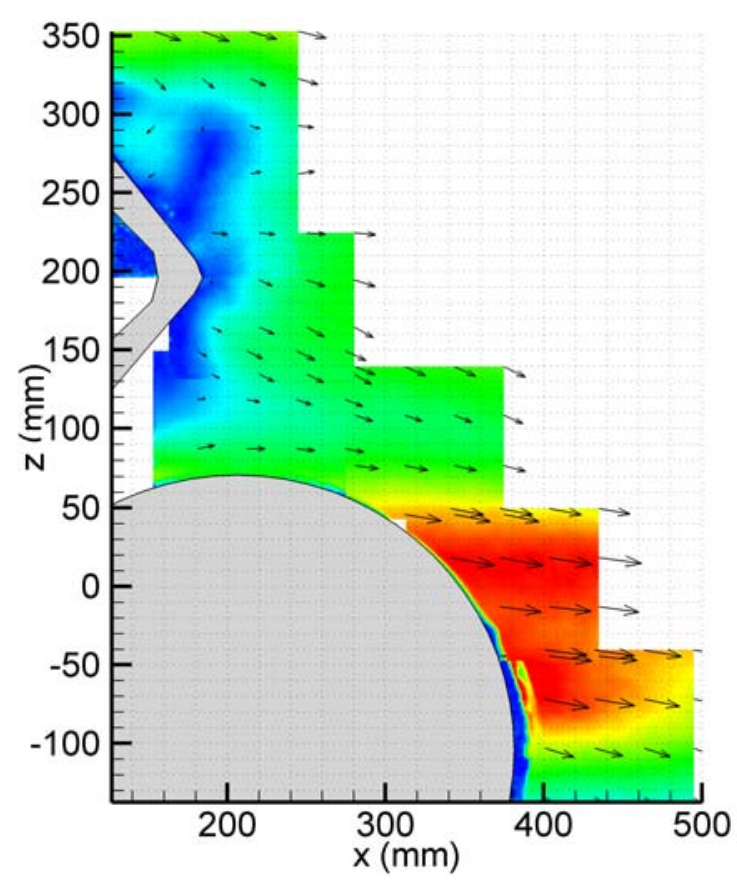

(a) LG

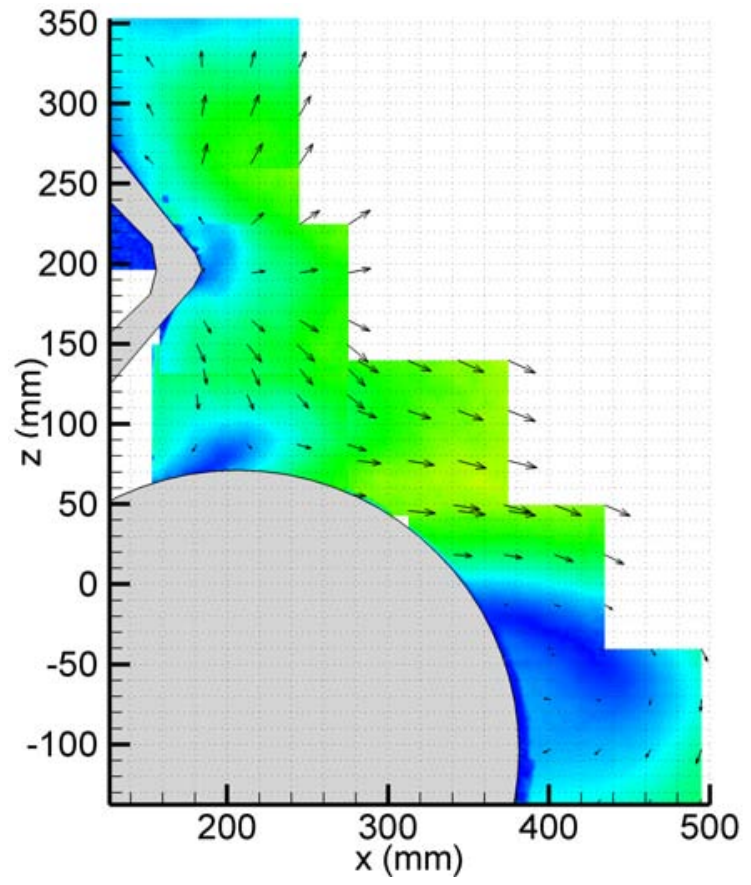

(b) Solid

Figure 8. Time-averaged velocity contours in the $x z-p l a n e$ at $\mathbf{y}=\mathbf{0}$ (torque link area, $U_{\infty}=40 \mathrm{~m} / \mathbf{s}$ ).

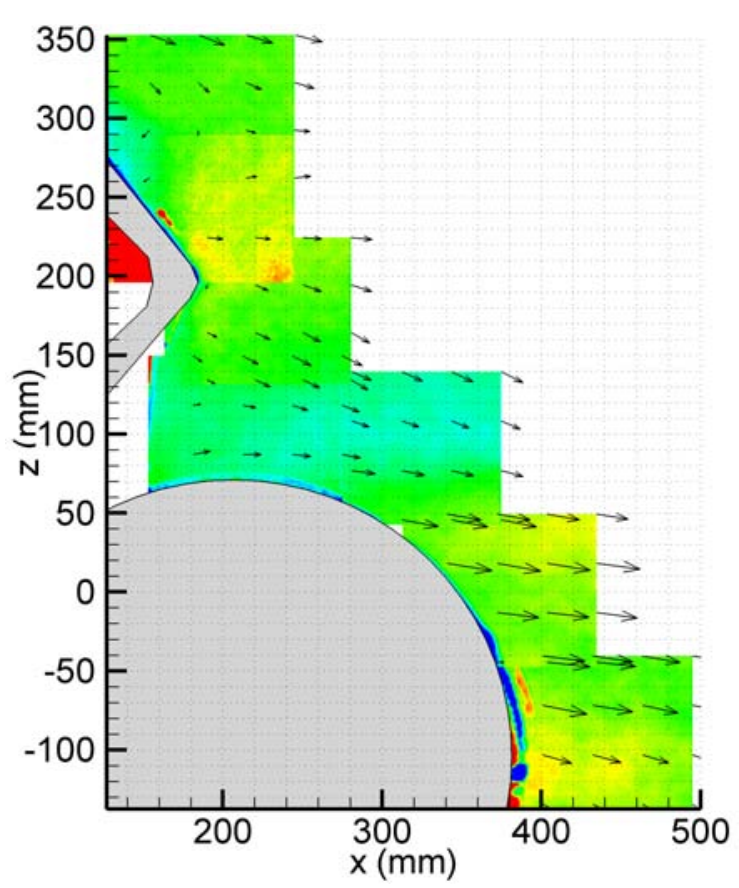

(a) LG

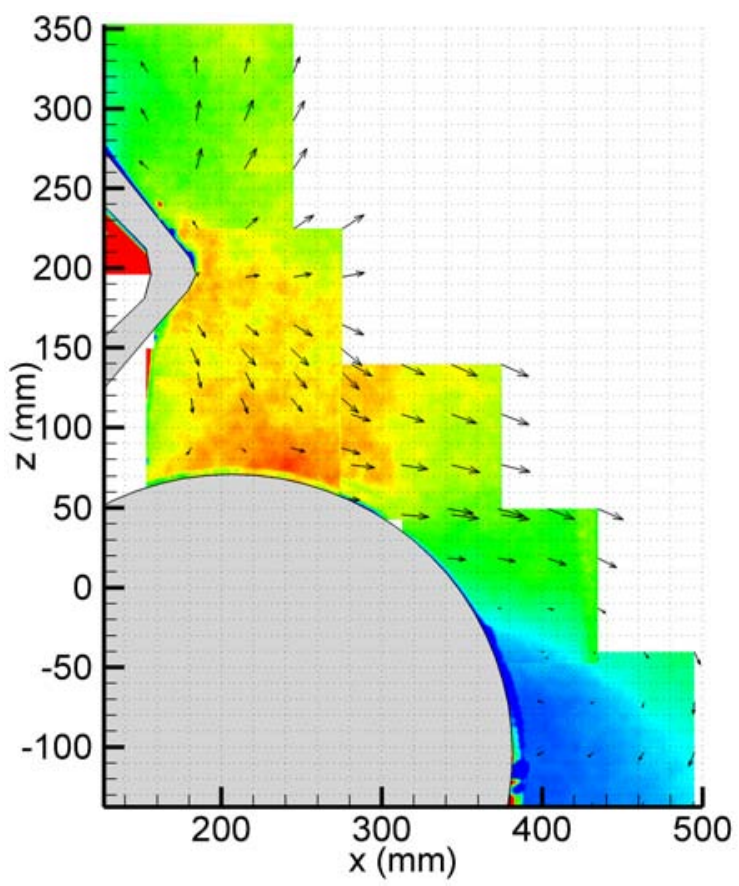

(b) Solid

Figure 9. Contours of velocity standard deviation in the xz-plane at $\mathbf{y}=\mathbf{0}$ (torque link area, $\left.U_{\infty}=40 \mathrm{~m} / \mathrm{s}\right)$. 
The wake structures of the solid and perforated fairings configurations are very similar. To allow for a quantitative comparison between these configurations, the time averaged variables of these configurations are subtracted in Figure 10. Due to the similarity in wake structure,

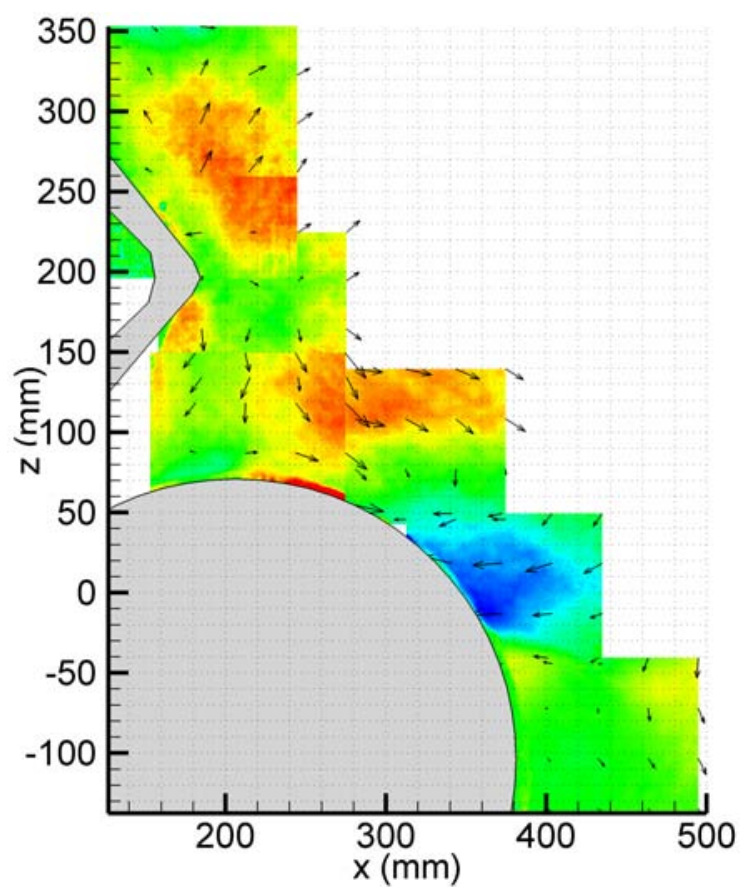

(a) Time averaged velocity

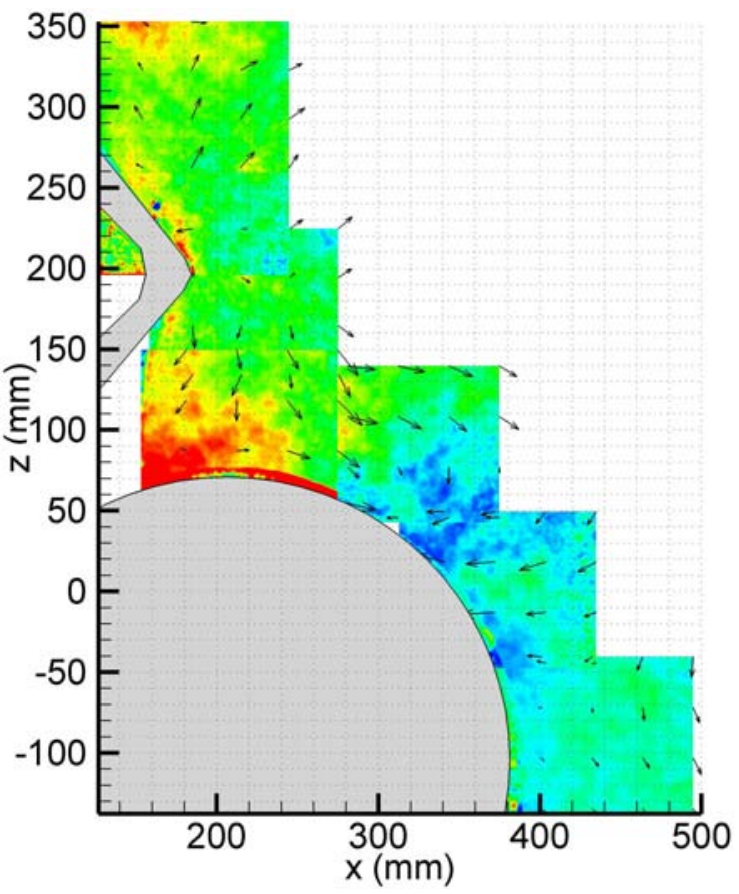

(b) Standard deviation of velocity

Figure 10. Difference between solid and perforated fairings for contours in the $x z-p l a n e$ at $y=0$ (torque link area, $\left.U_{\infty}=40 \mathrm{~m} / \mathbf{s}\right)$.

the direction of the subtracted vectors in Figure 10 aligns with the original flow direction in Figure $8(\mathrm{~b})$. Downstream of the torque link area between $\mathrm{z}=100$ and $\mathrm{z}=300 \mathrm{~mm}$, wake velocities are up to $30 \%$ lower for the perforated fairing. Plausibly, bleeding of air through the fairings reduces the high speed deflection past the fairing sides and thereby reduces wakes velocities in the centerplane. The earlier hypothesized large scale fluctuations associated with fairing size would then also reduce in magnitude. This can explain the decrease in turbulence intensity reflected in the standard deviation in Figure 10(b).

The velocity decrease in the torque link area is opposed to a velocity increase in the small jet between $\mathrm{z}=0$ and $\mathrm{z}=50 \mathrm{~mm}$. It is likely that the bled air through the undertray mixes with the flow that escapes from the undertray side edge and causes this velocity increase. Accompanied with the decrease of time averaged velocity comes a slight decrease in $u^{\prime}$ as depicted in the lower half of Figure 10(b).

Fluctuating surface pressures have been measured at several locations in the near wake (Figure 2). The data for location 1 and 5 are shown in Figure 11. The spectra in the back of the main leg are dominated by large scale turbulence and it's breakdown. With both solid and perforated fairings on, a hump is visible centered at $f \approx 104 \mathrm{~Hz}$. The frequency scales with freestream velocity and using the main leg diameter, the non dimensional frequency combines to $\operatorname{Str}=0.2$. The difference between solid and perforated fairings is small, although against expectations the perforated fairing exhibits slightly higher amplitude fluctuations. Without the fairings on, the hump disappears and the magnitude of the pressure fluctuations is significantly lower. This is combined with a $23 \%$ increase in base pressure to $C p=-0.79$. Although the freestream flow can directly impinge on the main leg at this z-coordinate, it seems that the vortex shedding is less intense than with fairings on. The fact that the application of the fairings enhances the vortex shedding at this location probably lies in the overlay between the upper articulation link fairing 


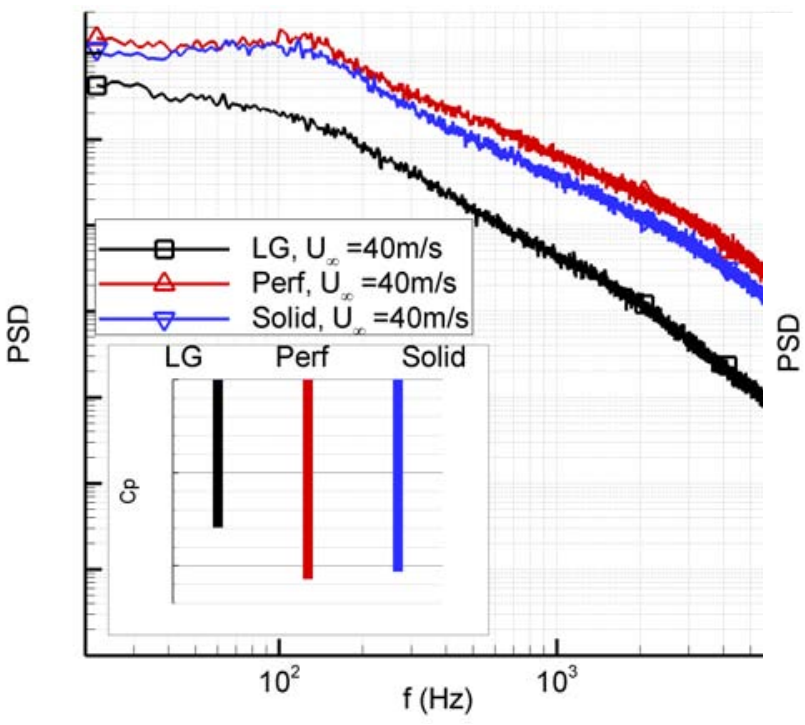

(a) Location 1, Main leg upper

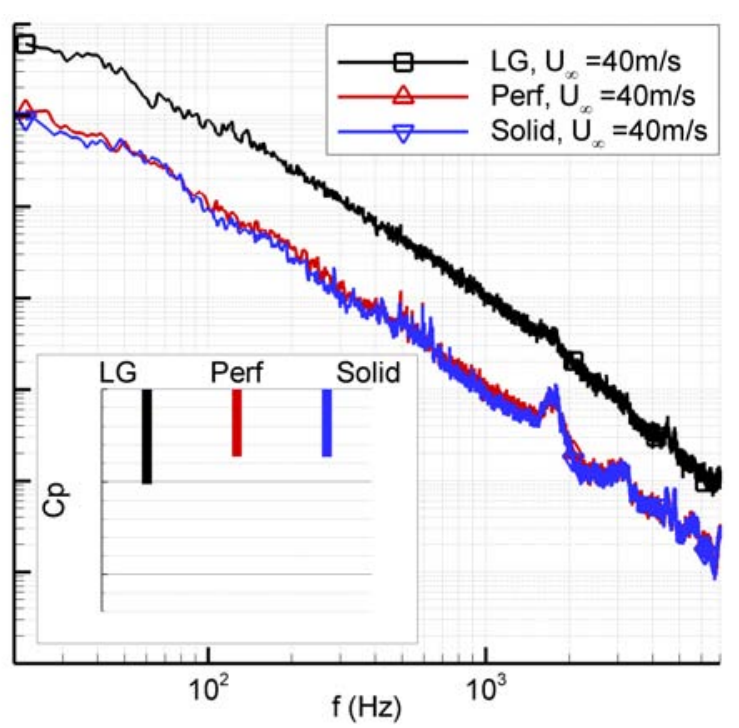

(b) Location 5, Rear bogie cap

Figure 11. PSD and mean surface pressure on the gear $\left(U_{\infty}=40 \mathrm{~m} / \mathbf{s}\right)$.

and the main leg, allowing the fairing boundary layer to flow past the sides of the main leg and separate from it.

The sensor in the rear bogie cap shows the inverse image of the sensor in the upper main leg. The PIV-measurements revealed that shielding of the bogie beam by the undertray results in a dead flow area at the rear of the bogie beam. Therefore fluctuations are of lower amplitude and base pressure is higher compared to the configuration without fairings. The difference between solid and perforated fairings is small for both unsteady and steady pressures.

\section{V.C. Wheel Flow}

The flow around the landing gear wheels are investigated using (un)steady pressure measurements. A local polar coordinate system can be defined originating in the wheel axis to describe location of the centerline tappings. The polar angle $\varphi$ is defined in the inset in Figure 12 and starts at the most upstream wheel position.

\section{V.C.1. Time averaged pressures}

The centerline tappings show few difference between the configurations in Figure 12. The front wheel exhibits separated flow roughly between $\varphi=90^{\circ}$ and $\varphi=270^{\circ}$, similar for all three configurations. The average level of the base pressure differs slightly, the LG configuration exhibiting the highest value. The base pressure is not constant and shows a dip at $\varphi=190^{\circ}$ with an amplitude of about $\Delta C p \approx 0.3$ for all configurations.

The rear wheel is showing a similar distribution to the front wheel in Figure 12(b). The $33^{\circ}$ inclination of the bogie beam with respect to the freestream flow direction displaces the wheels and prevents the wake of the front wheel from impinging directly on the rear wheel. There are few differences between the configurations, apart from the LG configuration exhibiting a $35 \%$ higher base pressure level. Base pressures are more constant in comparison to the front wheel. Also, suction is less on the lower wheel side $\left(\varphi>270^{\circ}\right)$ illustrated by a minimum value of $C p=-0.8$ opposed to $C p=-1.1$ for the front wheel. Additionally, the point of minimum pressure on the upper wheel side shifts from $\varphi=90^{\circ}$ to $\varphi=70^{\circ}$, advancing the separation point.

The centerline mean pressure signatures of both front and rear wheels indicate that perforating the fairings is not significantly altering the time averaged flow around the wheels. The results from the shoulder taps confirm this image and are omitted here. 


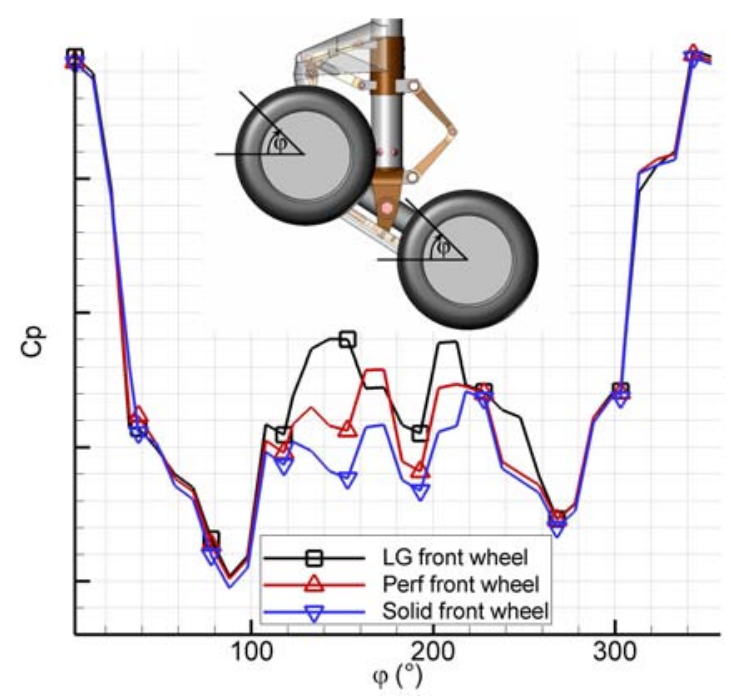

(a) Front wheel

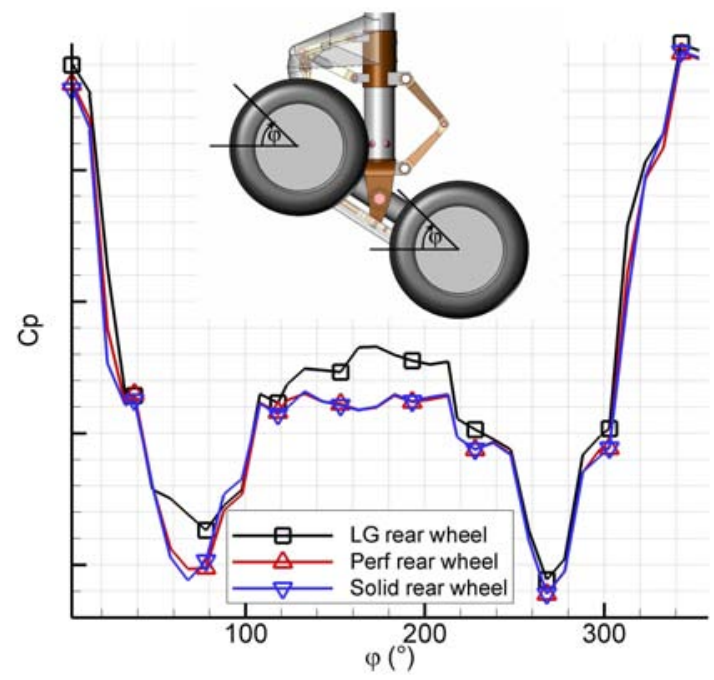

(b) Rear wheel

Figure 12. Centerline surface pressures on landing gear wheels $\left(U_{\infty}=40 \mathrm{~m} / \mathrm{s}\right)$.

\section{V.C.2. Unsteady pressures}

The PSD of the fluctuating pressures are shown in Figure 13 for several angular stations. The landing gear configurations without fairings, with perforated fairings and solid fairings are indicated using black, red and blue color respectively. The front and rear wheels can be distinguished by a dashed and solid line respectively. For the attached flow regions at $\varphi=348^{\circ}$ and $\varphi=303^{\circ}$, the PSD exhibits a low value since large amplitude fluctuations are not present. There is very few difference between the various configurations and the front and rear wheel. A low frequency tone is present at $f=48 \mathrm{~Hz}$. The spectral content of the tone is found to scale linear with velocity, implying the physical background is related to a shedding phenomenon. For the other angular stations, this low frequency tone is swamped by separation induced pressure fluctuations. The high frequency content for the attached flow regions is dominated by peaks in the PSD due to electronic noise.

For the front wheel, the unsteady pressures for the solid and perforated fairing at $\varphi=33^{\circ}$ and $\varphi=78^{\circ}$ show a similar signature to the PSD's at $\varphi=348^{\circ}$ and $\varphi=303^{\circ}$. However, the rear wheel pressures show a low frequency hump centered roughly around $f=125 \mathrm{~Hz}$, most pronounced for the solid fairing configuration. Application of perforations reduces the peak level of the fluctuations and shifts the center slightly upward to $f=150 \mathrm{~Hz}$. The absence of this phenomenon for the front wheel and the proximity of the angular stations to the front wheel points towards the unsteady front wheel wake responsible for the pressure fluctuations. However, the fact that the low frequency hump is hardly there for the plain landing gear configuration could also point in the direction of the undertray side edge vortex (visualized as nr.3 in Figure 7) impinging on the rear wheel tyre surface. The linear scaling of the humps with freestream velocity confirms the physical background of the phenomenon.

The high level of the PSD's for the rear wheel at $\varphi=78^{\circ}$ agrees with the forward movement of the separation point between the front and rear wheel as measured in the centerline mean pressure distribution in Figure 12. The stations at $\varphi=123^{\circ}, \varphi=168^{\circ}$ and $\varphi=213^{\circ}$ are located in the separated flow region, reflected by the level increase of the PSD. The fairing configurations appear to exhibit more unsteadiness than the landing gear configuration. The difference between porous or solid fairings is small. At $\varphi=258^{\circ}$, the flow is on the verge of separation at the underside of both rear and front wheel, explaining the level decrease of the PSD. As for the attached flow regions at $\varphi=348^{\circ}$ and $\varphi=303^{\circ}$, the differences between configurations are small here.

Averaging the unsteady pressures yields the mean pressures around the wheel circumference. 


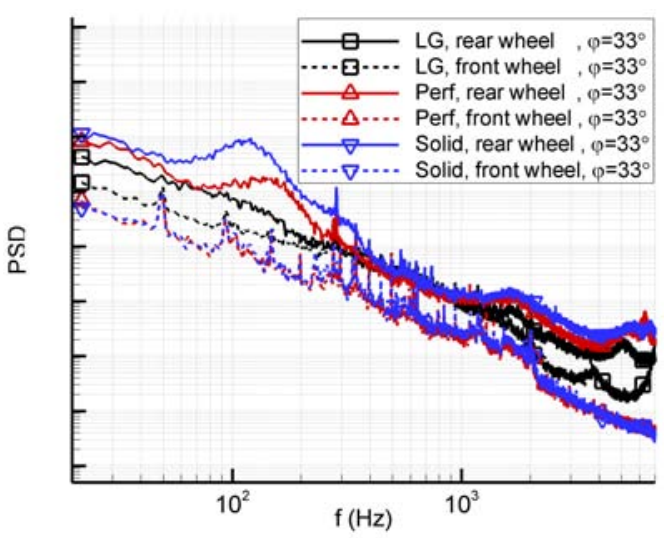

(a) $\varphi=33^{\circ}$

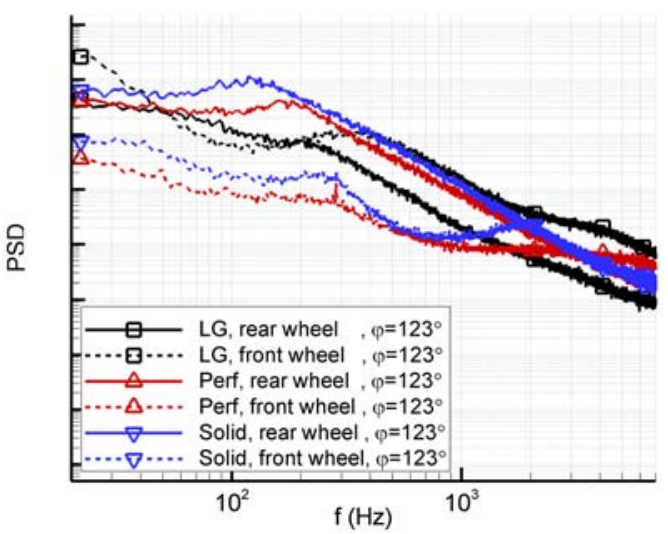

(c) $\varphi=123^{\circ}$

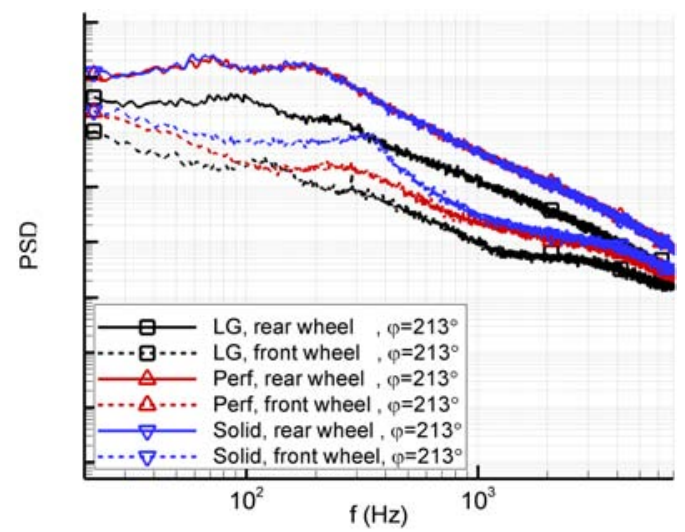

(e) $\varphi=213^{\circ}$

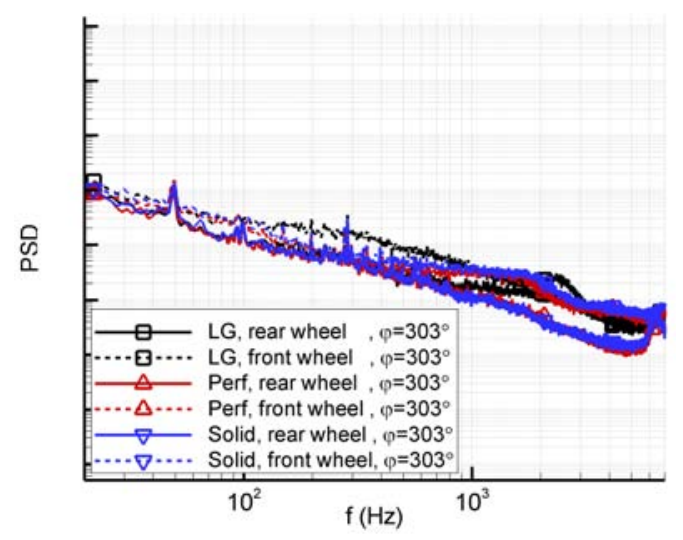

(g) $\varphi=303^{\circ}$

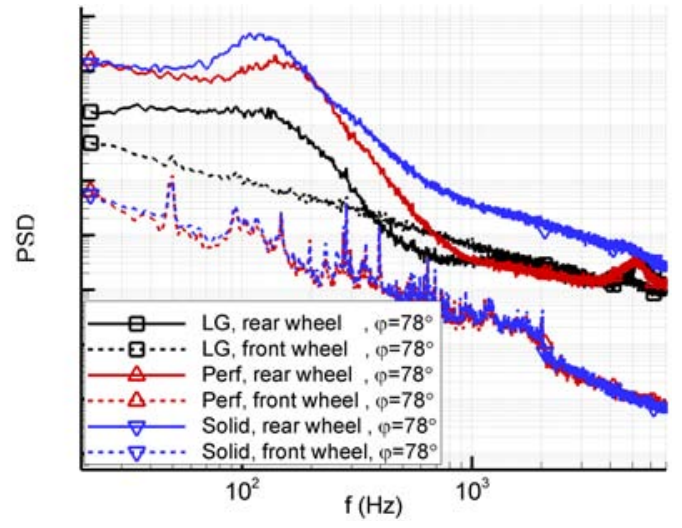

(b) $\varphi=78^{\circ}$

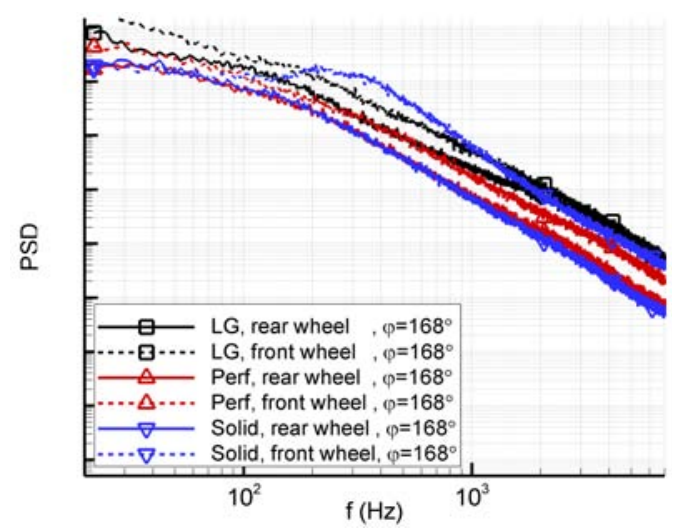

(d) $\varphi=168^{\circ}$

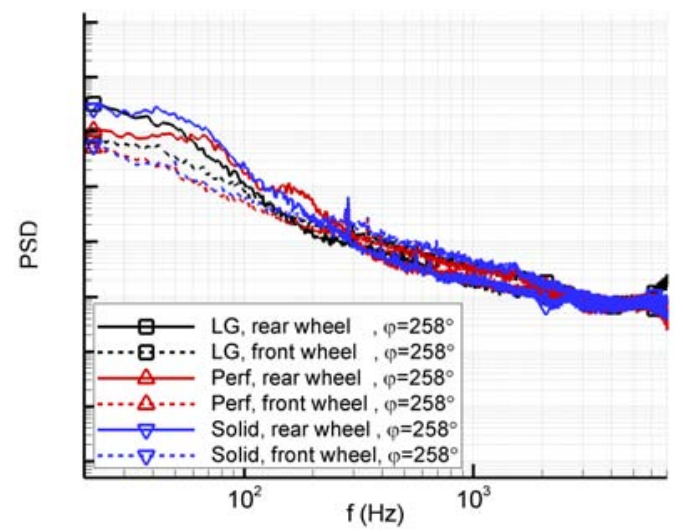

(f) $\varphi=258^{\circ}$

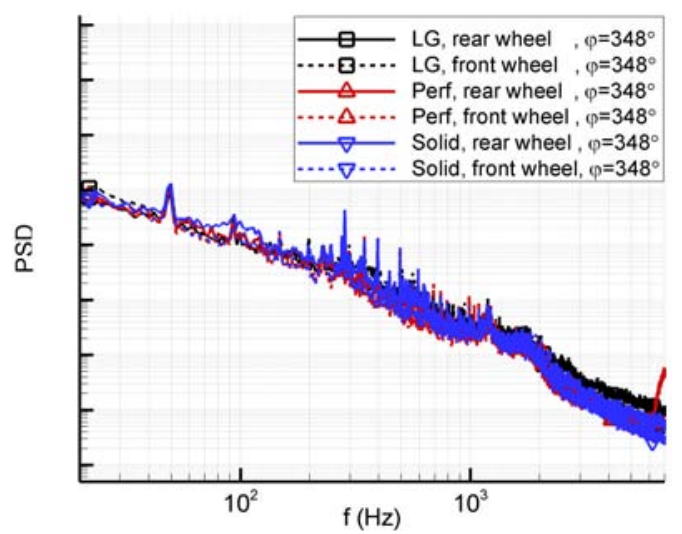

(h) $\varphi=348^{\circ}$

Figure 13. PSD of surface pressure on landing gear wheels $\left(U_{\infty}=40 \mathrm{~m} / \mathrm{s}\right)$. 
The obtained centerline pressure distributions in steps of $\Delta \varphi=45^{\circ}$ largely agree with the centerline pressure distributions as measured by the tappings discussed in section V.C.1.

\section{Acoustics}

The noise of the landing gear model has been investigated using microphone arrays and onsurface microphones.

\section{VI.A. Microphone array}

Beamforming plots centered at $f=2 \mathrm{kHz}$ for the different configurations are shown in Figure 14. The results reveal a decrease in noise source strength for this particular frequency by application

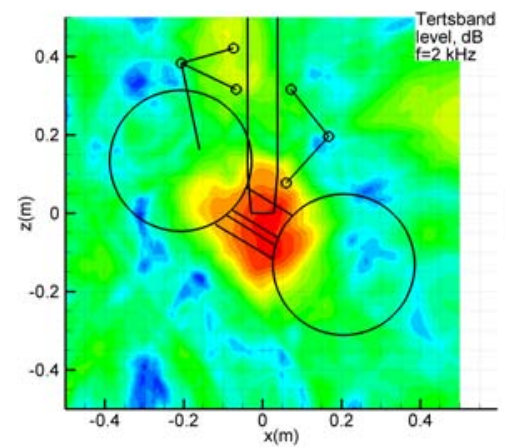

(a)

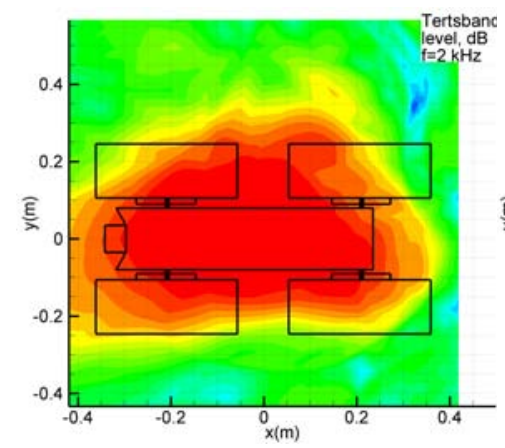

(d) LG

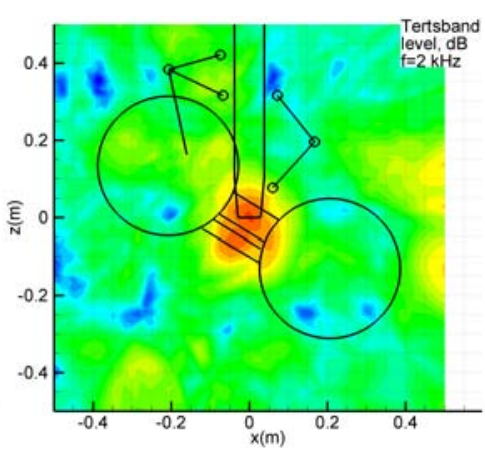

(b)

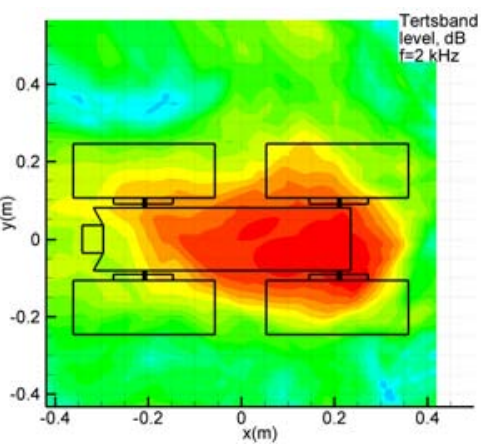

(e) Solid

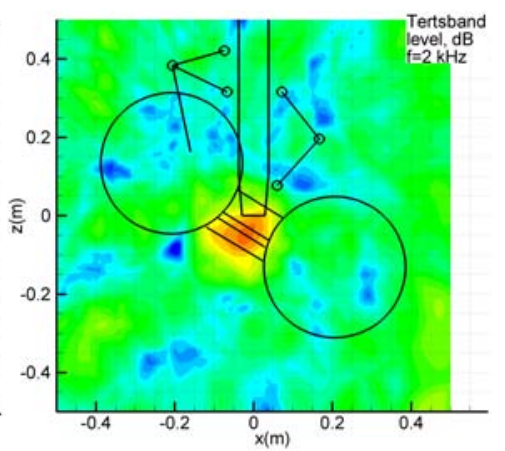

(c)

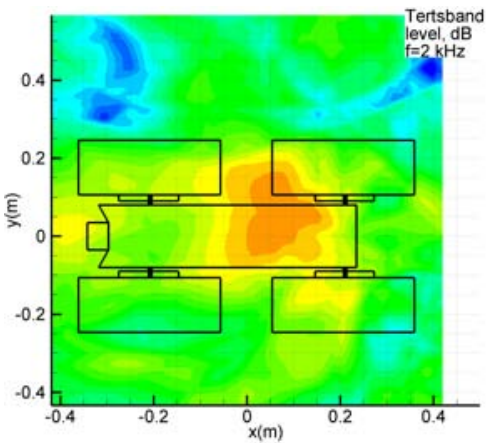

(f) Perf

Figure 14. Averaged beamforming plots centred at $2 \mathrm{kHz}$ from ceiling array (above) and side wall array (below) $\left(U_{\infty}=40 \mathrm{~m} / \mathbf{s}\right)$.

of perforated fairings. From the ceiling view, the main source is located between the wheels and remains there for the different configurations. The ground view from the side wall array shows the noise source reduce in size and move downstream with solid fairings applied. The source further diminishes in strength by application of the perforations.

At higher frequencies the resolution becomes better. Figure 15 shows clearly discernible sources centered at $10 \mathrm{kHz}$. From the ceiling view perspective, the solid fairing configuration is the most quiet for this frequency, showing an almost uniform background noise level. Application of perforations gives perforate noise on the upper articulation link fairing, elevating the background noise level considerably. Without fairings a noise source emerges between upper articulation link and the main leg. Additionally, a noise source appears at the brake rod junction with the yoke, plausibly because this item is not shielded anymore by the undertray. The location is confirmed by the ground view localization plot for this configuration in Figure 15(d). Shielded by the wheels for the ceiling view, noise sources emerge at the brake discs for the ground view. Application of fairings reduces the impingement velocity on the brake discs and hence the source 


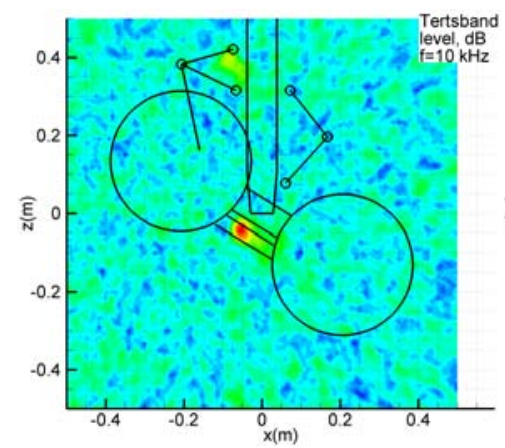

(a)

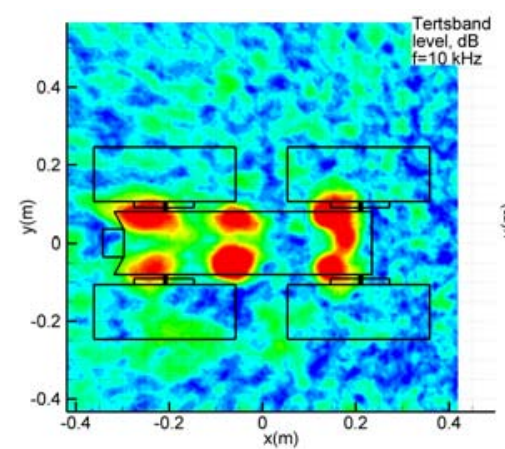

(d) LG

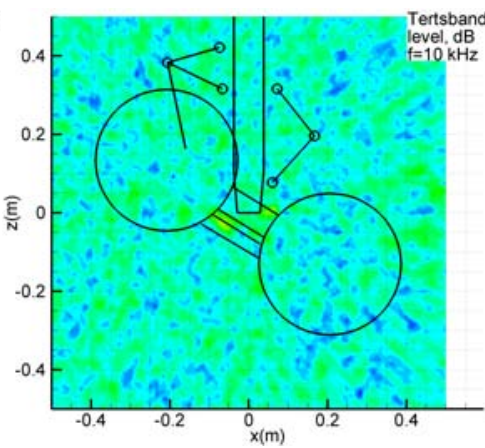

(b)

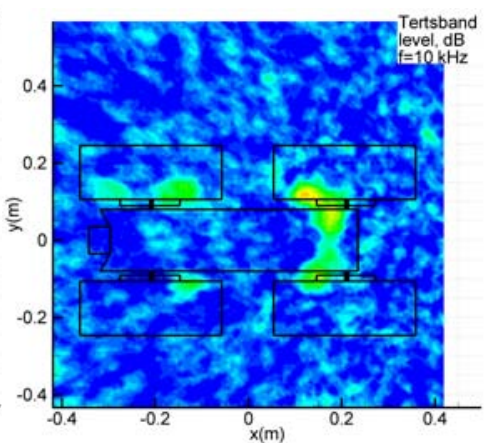

(e) Solid

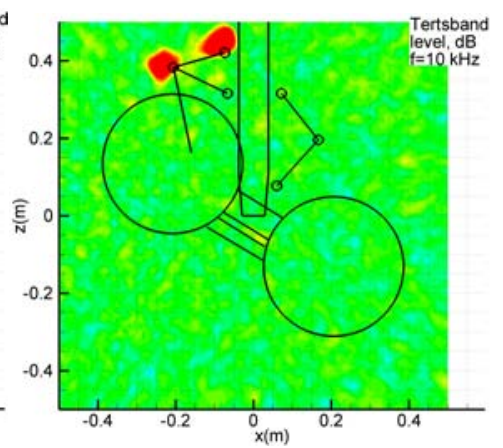

(c)

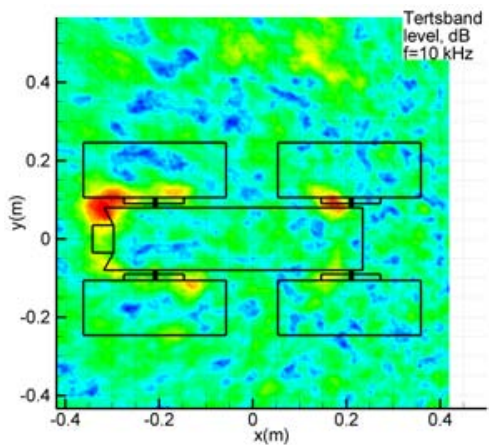

(f) Perf

Figure 15. Averaged beamforming plots centred at $10 \mathrm{kHz}$ from ceiling array (above) and side wall array (below) $\left(U_{\infty}=40 \mathrm{~m} / \mathrm{s}\right)$.

strength decreases, especially for the front breaks. The perforated fairings in Figure 15(f) show the perforate noise again, together with the elevated background noise level. These results clearly show that especially for high frequencies, shielding sharp objects from high speed impingement yields considerable noise reduction.

In order to compare the results over the frequency domain, an area integration is performed over the different beamforming plots. For the ceiling array, values are added in the rectangle between $x= \pm 0.2 \mathrm{~m}$ and $z=-0.1 \mathrm{~m}$ to $z=0.3 \mathrm{~m}$. For the side wall array, the rectangle comprises of $x=-0.4 \mathrm{~m}$ to $x=0.3 \mathrm{~m}$ and $y= \pm 0.15 \mathrm{~m}$. By covering various parts of the perforated fairings with tape, a study is performed on the effectiveness of perforation location. The undertray and articulation link stagnation area are deduced from the oil flow visualization in Figure 4.

A comparison showing the effect of the main configurations referenced to the solid fairing configuration is illustrated in Figures 16(a) and 16(b) for ceiling and side wall array respectively. The ceiling array shows a noise decrease by application of solid fairings in the mid frequency range and above $f=10 \mathrm{kHz}$. Lower frequencies (below $f=600 \mathrm{~Hz}$ ) are noisier, but application of perforations makes this low frequency increase disappear. However, exposing all perforations shows perforate noise emerging above $f=8 \mathrm{kHz}$. By exposing only the stagnation area of the perforations, the low frequency decrease persists while the perforate noise disappears. The side wall array corresponding to the ground view shows a similar trend. The plain landing gear configuration is noisier above $f=1 \mathrm{kHz}$ instead of the smaller frequency range for the ceiling array. The dip for all presented configurations at the $f=500 \mathrm{~Hz}$ band indicates a sharp noise increase in this band for the referenced solid fairing configuration. Although the limited resolution of the beamforming plots at this frequency hamper source localization, inspection of the plots reveals a large noise source at the gear for the solid configuration opposed to wake noise sources for the plain landing gear and perforated fairing configuration. 


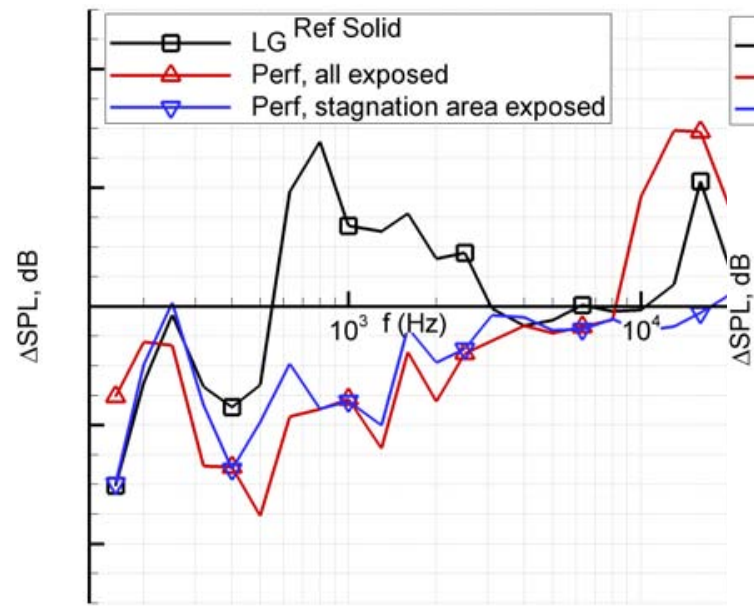

(a) Effect of solid and perforated fairings

Ceiling array

$$
\begin{aligned}
& \text { Averaged between } x= \pm 0.2 \mathrm{~m} \text { and } z=-0.1 \mathrm{~m} \text { to } \\
& z=0.3 \mathrm{~m}
\end{aligned}
$$

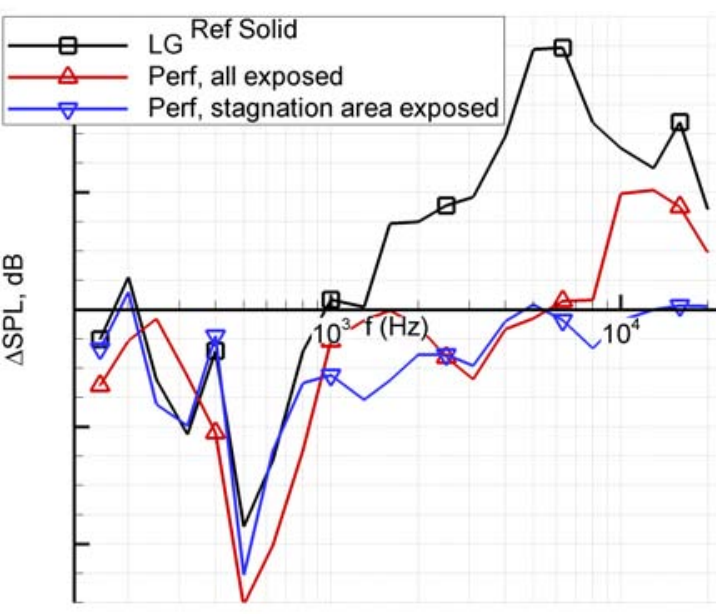

(b) Effect of solid and perforated fairings

Wall array

$$
\begin{aligned}
& \text { Averaged between } x=-0.4 \mathrm{~m} \text { to } x=0.3 \mathrm{~m} \text { and } \\
& y= \pm 0.15 \mathrm{~m}
\end{aligned}
$$
$y= \pm 0.15 \mathrm{~m}$

Figure 16. Comparison of levels from integrated beamforming plots (referenced to Solid configuration, $\left.U_{\infty}=40 \mathbf{m} / \mathbf{s}\right)$.

\section{VI.B. On-surface microphones}

The narrowband spectra resulting from the on-surface microphone measurements for various locations (Figure 2) are shown in Figure 17. The microphones in location 1 and 2 in the back of the strut are swamped by the large scale velocity fluctuations impinging on the back of the strut. Therefore acoustic phenomena are difficult to observe in these spectra and difference between configurations is hardly discernible. Unsteady pressure sensors are less sensitive than microphones, hence the PSD in Figure 11(a) for location 1 shows a different image to the narrowband spectrum in Figure 17(a).

At the lower main leg location, the LG configuration shows a hump at $f \approx 104 \mathrm{~Hz}$ or Str $\approx 0.2$ based on freestream velocity and leg diameter. It is believed to correspond to vortex shedding noise from the main leg. Since the fairings upstream of this part of the strut prevent high speed impingement on the main leg, the hump is not visible with the fairings applied. Also, a peak corresponding to perforate noise is visible just above $f=10 \mathrm{kHz}$ with all perforations exposed. The peak scales linearly with freestream velocity $U_{\infty}$. The perforate noise is also picked up by the microphones in locations 3 and 5. A localization study utilizing tape is carried out for the microphone measurements. It reveals the sides of the lower articulation link being responsible for the perforate noise peak just above $f=10 \mathrm{kHz}$. This is conform to results in previous literature,${ }^{6}$ which already showed the shearing flow past the perforate is causing most of the noise. Comparing the non-dimensional frequency (referenced to local velocity and orifice diameter $d_{o r}$ ) with these experiments is difficult since the local velocity in the present experiment is unknown. Estimating this variable at 1.5 times the freestream velocity $U_{\infty}$ yields agreement between the Strouhal numbers for both experiments at $S t r_{d_{o r}}=0.33$.

The spectra of the landing gear configuration at location 3 and 5 exhibit a higher level compared to when the fairings are applied. Flow noise at the microphones is responsible for this higher level, since application of fairings prevents high speed flow in these areas. A clear difference between solid and perforated fairings is noticeable at location 3 above $f=100 \mathrm{~Hz}$ and almost up to $f=10 \mathrm{kHz}$. This elevated level of the solid fairing configuration in the low to mid frequency range agrees with the integrated wall- and ceiling array beamforming plot levels in Figure 16. Subtracting the tertsband averaged levels from this microphone further clarifies the difference between the configurations in Figure 18. A comparison to different freestream velocity values shows the frequency content to scale linearly with velocity. Additionally, the difference in magnitude 


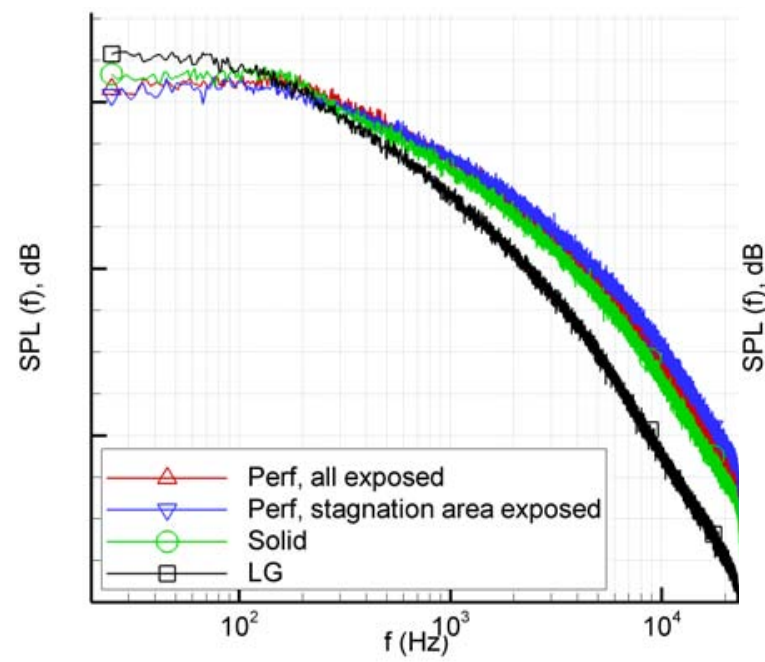

(a) Location 1, Main leg upper

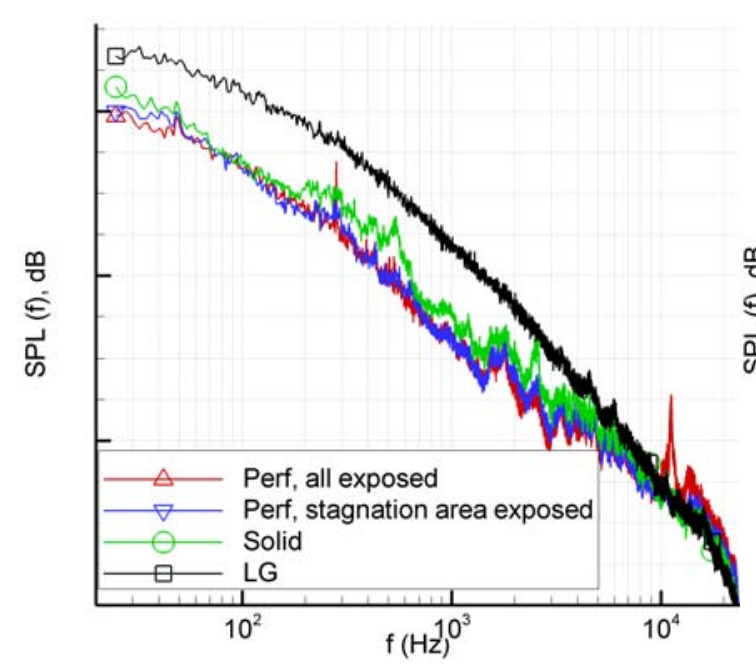

(c) Location 3, Bogie-articulation link junction

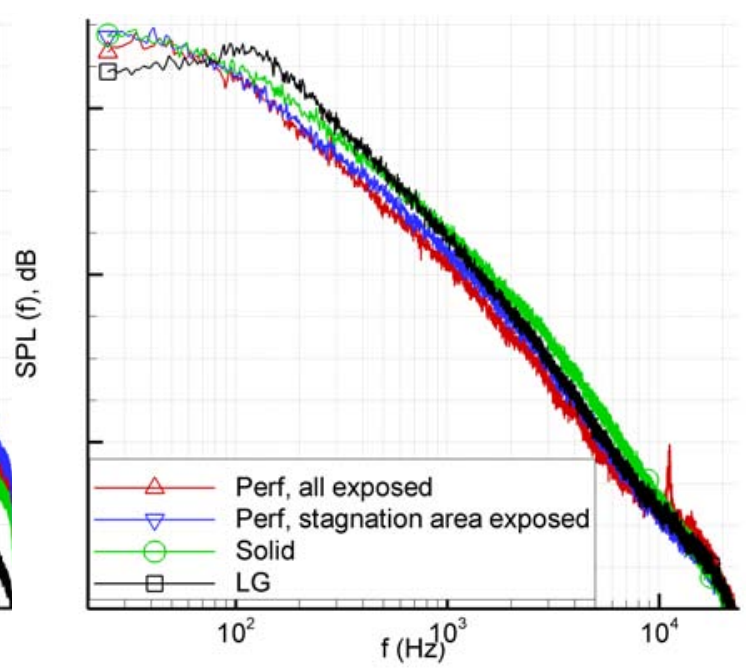

(b) Location 2, Main leg lower

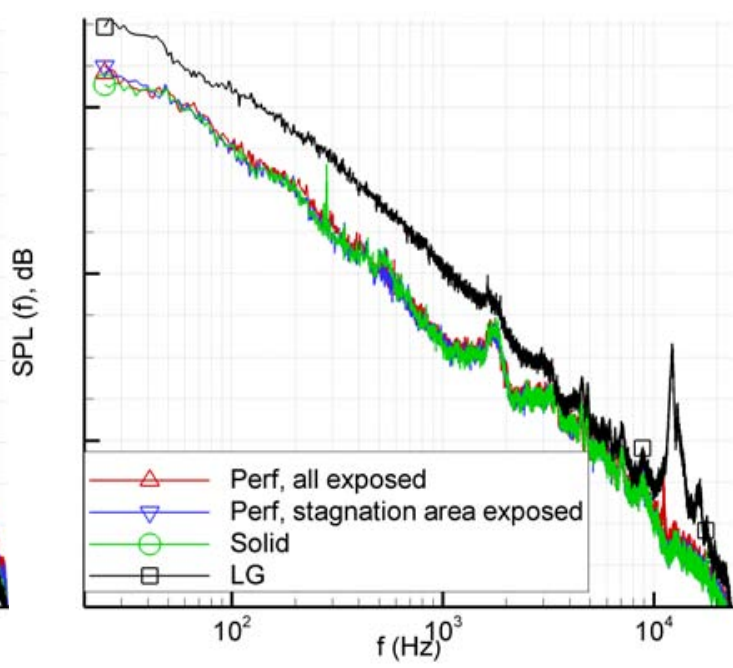

(d) Location 5, Rear bogie cap

Figure 17. Narrowbandspectra of on surface microphones on the gear $\left(U_{\infty}=40 \mathrm{~m} / \mathrm{s}\right)$. 


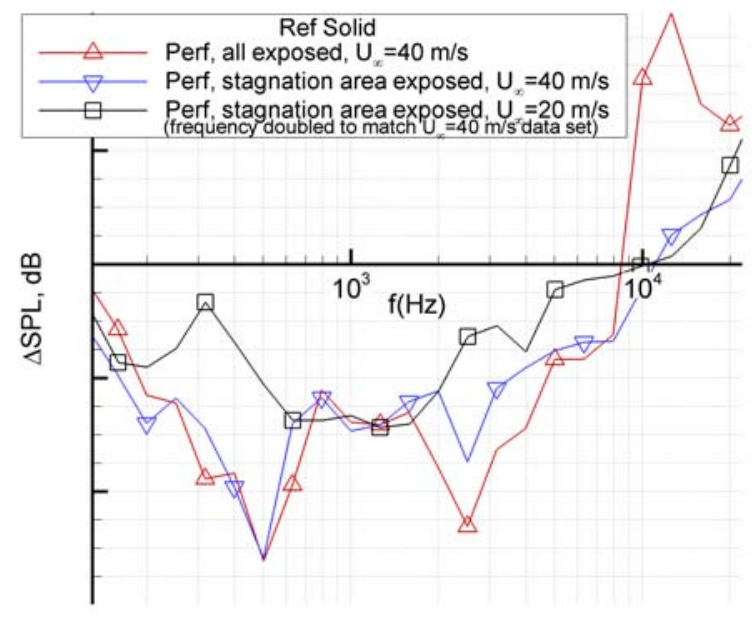

Figure 18. Subtracted tertsband averaged levels from microphone at bogie-artlink junction (referenced to Solid configuration).

between both configuration increases with freestream velocity. Interaction of the separated flow from the fairings with the downstream gear components is believed to be responsible for the elevated noise level above $f=100 \mathrm{~Hz}$. In this frequency range, the solid configuration displays a peak at $f=560 \mathrm{~Hz}$ which is believed to correspond to the dip in the integrated side wall array levels in Figure 16. The perforation location effectiveness study utilizing tape shows the articulation link fairing responsible for the noise. However, the peak stands out better in the farfield wall array measurements than the nearfield on-surface microphone results.

Above $f=1000 \mathrm{~Hz}$, the spectra in the dead flow areas (location 3 and 5) are far from smooth and electronic noise is dominant. Hence the several peaks in this part of the narrowband spectra show no scaling with freestream velocity and are not believed to represent physical phenomena. The difference between all perforations exposed or only the stagnation point area perforations is hardly noticeable, except from the occurrence of the perforate noise above $f=10 \mathrm{kHz}$.

\section{Conclusion}

An aerodynamic and acoustic survey has been performed on a simplified A340 look alike Main Landing Gear to explore the influence of (perforated) fairings on the lower part of the gear.

The oil flow visualization combined with the PIV have clarified flow direction and magnitude around the fairings and in the wake centerplane. The undertray fairing exhibits largely attached flow and divides the airstream in up- and downward direction. The downward pushed flow partly escapes between front and aft wheels and produces a side edge vortex on the undertray side. The shielding of the bogie beam results in a dead flow area directly aft of the rear bogie end. The upward directed flow forms a horseshoe vortex around the lower articulation link fairing and pushes itself further upward on the upper articulation link fairing. The application of the fairings increases the frontal area and therefore bends the flow in transverse and vertical direction, preventing the airstream from penetrating in streamwise direction past the gear components. This influences the wake structure and hence the turbulence levels in the proximity of the downstream gear components. The application of the perforations does not significantly alter the flow structure in the wake or in front of the fairings, indicated by equal flow directionality between solid and perforated configurations. However, flow velocity and turbulence levels in the near wake of the torque link are lower than for impermeable fairings. It is hypothesized that by bleeding air, large scale unsteadiness associated with the fairings decreases resulting in this reduction.

The flow around the wheels is studied by means of centerline and shoulder pressure taps on the tyre surface. The mean surface pressures show few difference between the various configurations, 
indicating that the time averaged flow around the wheels is largely unaltered by application of (perforated) fairings. The unsteady surface pressures show increased unsteadiness on the upper side of the rear wheels with fairings applied, pointing towards the undertray side edge vortex and/or the front wheel wake impinging on the rear tyre surface.

Upward from the torque link, unsteady pressure sensor measurements indicate that application of the fairings enhances the vortex shedding from the main leg at this position. By engineering judgement the overlay between upper articulation link fairing and the main leg is responsible for the enhanced shedding. Downward from this position the fairings prevent high velocities impinging on the main leg. Therefore vortex shedding features at this position of the main leg are only observed without fairings applied. The aerodynamic tests indicate that more porosity is needed to induce more significant changes to the flow field by perforating the fairings.

The acoustics are studied employing several on-surface microphones and two microphone arrays to measure the ground- and side view noise signature. For both directions, application of solid fairings has been shown to reduce the noise in the mid and high frequency domain compared to the plain landing gear configuration up to $4.5 \mathrm{~dB}$. However a noise increase is measured in the low frequency domain. The application of perforations reduces the low frequency noise introduced by the solid fairings to values below the plain landing gear configuration for both arrays. Additionally, reduced levels are measured in the mid frequency domain by application of the perforations. The low to mid frequency noise reduction is confirmed by measurements of an on-surface microphone located in the bogie beam - articulation link junction. Combined with the linear velocity scaling of the noise, this points in the direction of large scale separation of the fairings interacting with the downstream gear components responsible for this phenomenon.

To investigate the effectiveness of perforation location, various parts of the fairing surfaces have been covered with tape. It appears that the stagnation area perforations are responsible for most of the noise decrease, for both the articulation link and undertray fairing. Agreeing with basic experiment results, most of the perforate noise is emitted from the lower articulation link sides at a Strouhal number of $S t r=0.4$ based on orifice diameter $d_{o r}$ and local velocity .

\section{Acknowledgements}

Special thanks go to Matthew Spiteri and Mike Tudor-Pole for their assistance during the wind tunnel experiments. Their support is greatly appreciated.

\section{References}

${ }^{1}$ Dobrzynski, W. and Buchholz, H., "Full-Scale Noise Testing on Airbus Landing Gears in the German Dutch Wind Tunnel," AIAA Paper 97-1597, 1997.

${ }^{2}$ Dobrzynski, W., Chow, L., Guion, P., and Shiells, D., "A European Study on Landing Gear Airframe Noise Sources," AIAA Paper 2000-1971, 2000.

${ }^{3}$ Piet, J.-F., Chow, L., Laporte, F., and Remy, H., "Flight Test Investigation of High-Lift Devices and Landing Gear Modifications to Achieve Airframe Noise Reduction," ECCOMAS 2004 conference, Jyvaeskylae, Finland, July 2004.

${ }^{4}$ Smith, M., Fenech, B., Chow, L., Molin, N., Dobrzynski, W., and Seror, C., "Control of Noise Sources on Aircraft Landing Gear Bogies," AIAA Paper 2006-2626, 2006.

${ }^{5} \mathrm{Li}$, Y., Smith, M., Zhang, X., and Molin, N., "Noise Sources Control of an Aircraft Landing Gear," AIAA Paper 2007-3465, 2007.

${ }^{6}$ Boorsma, K., Zhang, X., and Molin, N., "Perforated Farings for Bluff Body Noise Control," AIAA Paper 2007-3462, 2007.

${ }^{7}$ Smits, A. and Lim, T., Flow visualization: techniques and examples, Imperial College Press, 2000.

${ }^{8}$ Raffel, M., Willert, C., and Kompenhans, J., Particle Image Velocimetry, A Practical Guide, Springer-Verlag, corrected 3rd printing ed., 2000.

${ }^{9}$ Moffat, R., "Describing the Uncertainties in Experimental Results," Experimental Thermal and Fluid Science, Vol. 1, 1988, pp. 3-17.

${ }^{10}$ Fenech, B. and Takeda, K., "SotonArray: Southampton University Wind Tunnel Microphone Array System Guide," Tech. Rep. AFM 07/03, University of Southampton, School of Engineering Sciences, 2007. 